\title{
Active Dendrites Regulate Spectral Selectivity in Location-Dependent Spike Initiation Dynamics of Hippocampal Model Neurons
}

\author{
Anindita Das and Rishikesh Narayanan \\ Cellular Neurophysiology Laboratory, Molecular Biophysics Unit, Indian Institute of Science, Bangalore 560012, India
}

\begin{abstract}
How does the presence of plastic active dendrites in a pyramidal neuron alter its spike initiation dynamics? To answer this question, we measured the spike-triggered average (STA) from experimentally constrained, conductance-based hippocampal neuronal models of various morphological complexities. We transformed the STA computed from these models to the spectral and the spectrotemporal domains and found that the spike initiation dynamics exhibited temporally localized selectivity to a characteristic frequency. In the presence of the hyperpolarization-activated cyclic nucleotide-gated (HCN) channels, the STA characteristic frequency strongly correlated with the subthreshold resonance frequency in the theta frequency range. Increases in $\mathrm{HCN}$ channel density or in input variance increased the STA characteristic frequency and its selectivity strength. In the absence of $\mathrm{HCN}$ channels, the STA exhibited weak delta frequency selectivity and the characteristic frequency was related to the repolarization dynamics of the action potentials and the recovery kinetics of sodium channels from inactivation. Comparison of STA obtained with inputs at various dendritic locations revealed that nonspiking and spiking dendrites increased and reduced the spectrotemporal integration window of the STA with increasing distance from the soma as direct consequences of passive filtering and dendritic spike initiation, respectively. Finally, the presence of HCN channels set the STA characteristic frequency in the theta range across the somatodendritic arbor and specific STA measurements were strongly related to equivalent transfer-impedance-related measurements. Our results identify explicit roles for plastic active dendrites in neural coding and strongly recommend a dynamically reconfigurable multi-STA model to characterize location-dependent input feature selectivity in pyramidal neurons.
\end{abstract}

Key words: active dendrites; $\mathrm{HCN}$ channels; hippocampus; intrinsic plasticity; spike initiation dynamics; spike-triggered average

\section{Introduction}

Pyramidal neurons are prodigious computational devices that transform the complexity of tens of thousands of afferent synaptic inputs into action potentials that encode and transmit critical features about these inputs. Deciphering this transformation and unraveling the input features represented by these neurons constitute the crux of the conjoined problems of coding and dynamics in neuronal systems (Ermentrout et al., 2007; Famulare and Fairhall, 2010; Hong et al., 2012; Ratté et al., 2013). The spiketriggered average (STA), computed by averaging inputs that trigger spikes when neurons are presented with white noise inputs, has proven to be extremely useful in characterizing feature representation in sensory neurons (Rieke et al., 1999; Dayan and Abbott, 2005). Similar approaches using white noise currents to

Received July 28, 2013; revised Nov. 20, 2013; accepted Dec. 8, 2013.

Author contributions: A.D. and R.N. designed research; A.D. performed research; A.D. and R.N. analyzed data; A.D. and R.N. wrote the paper.

This work was supported by the International Human Frontier Science Program Organization, Department of Biotechnology (DBT), through the US-India Brain Research Collaborative Program and the DBT-Indian Institute of Science partnership program. We thank members of the cellular neurophysiology laboratory for helpful discussions and for critical comments on a draft of this manuscript.

Correspondence should be addressed to Rishikesh Narayanan, PhD, Molecular Biophysics Unit, Indian Institute of Science, Bangalore 560 012, India. E-mail: rishi@mbu.iisc.ernet.in.

DOI:10.1523/JNEUROSCI.3203-13.2014

Copyright $\odot 2014$ the authors $\quad 0270-6474 / 14 / 341195-17 \$ 15.00 / 0$ model synaptic inputs have been used to understand the biophysical mechanisms behind specific characteristics of singleneuronal spike initiation dynamics. These studies have provided us with valuable insights on the relationships between the form of STA and the class of neuronal excitability and on voltage-gated ion channels (VGICs) involved in governing specific aspects of spike initiation (Agüera y Arcas and Fairhall, 2003; Prescott et al., 2008a; Famulare and Fairhall, 2010; Ratté et al., 2013).

In analyzing single-neuron feature selectivity through the STA, a single linear kernel is generally deemed to adequately characterize the neuronal input-output transformation (to a first-order approximation) across all afferent inputs. However, pyramidal neurons are endowed with extensive dendritic arbor, defined by non-isopotential compartments that filter synaptic inputs differentially based on their location along the arbor (Rall, 1977; Magee, 2000; Williams and Stuart, 2003; Spruston, 2008). Furthermore, research spanning the past two decades has established unambiguously the existence of several VGICs distributed with nonuniform densities across the dendritic arbor (Migliore and Shepherd, 2002; Johnston and Narayanan, 2008; Nusser, 2012), adding an additional layer of complexity to the location dependence of input processing. Finally, a large body of accumulating evidence has demonstrated clearly that dendritic VGICs can undergo localized or spatially widespread plasticity in several 
of their properties, implying dynamic regulation of such location-dependent input processing (Magee and Johnston, 2005; Remy et al., 2010; Shah et al., 2010; Narayanan and Johnston, 2012).

Against this background of dynamically regulated, locationdependent processing by the plastic active dendrites of pyramidal neurons, we investigated whether a single, location-invariant input-output kernel could still characterize the spike initiation dynamics. To address this, we first developed specific measures related to the STA in the spectral and temporal domains so that we could assess their sensitivities to parametric variations quantitatively and relate them to subthreshold impedance properties. We used conductance-based neuronal models of various morphological complexities and systematically assessed the roles of dendrites, their VGICs, and their plasticity in altering these quantitative STA measures. Our results suggest that the presence of dendrites and their ion channels alter spike initiation dynamics in a location-dependent manner, with input statistics and the density, kinetics, and voltage dependence of the ion channels playing critical roles in qualitatively and quantitatively altering the nature of the STA. Finally, based on these results, we propose a dynamically reconfigurable, multi-STA model to represent neurons with extensive dendritic arborization characterized by plastic intrinsic properties.

\section{Materials and Methods}

Models

We used three different models to investigate the impact of dendrites and the active conductances therein on neuronal spiking dynamics. We began with a single compartment model to tease apart the effects of the specific active conductances and passive properties of a neuron on its STA. We then moved to a ball-and-stick model and finally to a morphologically realistic model to delineate the roles of dendrites and dendritic VGICs in regulating STA. Such staggered analyses spanning models of different arborizational complexities allowed us to perform systematic sensitivity analyses related to all model parameters while accounting for the confounding effects of interactions among these parameters. All of our models and their parameters were based on the large body of quantitative experimental data available on the morphology and the passive and active properties of hippocampal CA1 pyramidal neurons and their dendrites.

Single compartmental model. Our single compartmental model comprised a cylindrical soma of $100 \mu \mathrm{m}$ length and $100 \mu \mathrm{m}$ diameter, with specific membrane resistivity $R_{\mathrm{m}}$, specific intracellular resistivity $R_{\mathrm{a}}$, and specific membrane capacitance $C_{\mathrm{m}}$ set at $30 \mathrm{k} \Omega \cdot \mathrm{cm}^{2}, 100 \Omega \cdot \mathrm{cm}$, and 1 $\mu \mathrm{F} / \mathrm{cm}^{2}$, respectively. This basic model consisted of Hodgkin-Huxleytype sodium channels, delayed rectifier potassium (KDR) channels, and hyperpolarization-activated cyclic nucleotide-gated ( $\mathrm{HCN})$ channels. The voltage dependencies and kinetics for sodium and KDR potassium channels were adopted from experimental measurements on hippocampal pyramidal neurons (Magee and Johnston, 1995; Hoffman et al., 1997; Migliore et al., 1999). The default density of sodium $\left(\bar{g}_{\mathrm{Na}}\right)$ and KDR channels $\left(\bar{g}_{K D R}\right)$ were set at 30 and $0.6 \mathrm{mS} / \mathrm{cm}^{2}$ and reversal potentials to $55 \mathrm{mV}$ and $-90 \mathrm{mV}$, respectively. The baseline density of HCN channels was fixed at $100 \mu \mathrm{S} / \mathrm{cm}^{2}$ and the kinetics and voltage dependence of the current through HCN channels, $I_{\mathrm{h}}$, were adopted from (Magee, 1998; Poolos et al., 2002; Gasparini et al., 2004) as follows:

$$
I_{\mathrm{h}}=\bar{g}_{h} s\left(V_{\mathrm{m}}, t\right)\left(V_{\mathrm{m}}-E_{\mathrm{h}}\right)
$$

where $\bar{g}_{h}$ is the maximal HCN conductance, $V_{\mathrm{m}}$ the membrane voltage, and $E_{\mathrm{h}}$ the HCN-channel reversal potential, which was set at $-30 \mathrm{mV}$. $s\left(V_{\mathrm{m}}, t\right)$ controlled the voltage dependence and kinetics of the HCN channel and evolved based on first-order kinetics as follows:

$$
\frac{d s}{d t}=\frac{s_{\infty}-s}{\tau_{\mathrm{HCN}}}
$$

$$
\begin{aligned}
s_{\infty}\left(V_{m}\right) & =\left(1+\exp \left(\left(V_{m}-V_{1 / 2}\right) / 8\right)\right)^{-1} \\
\tau_{\mathrm{HCN}}\left(V_{m}\right) & =\frac{F_{\tau} \exp \left(0.033\left(V_{m}+75\right)\right)}{0.011\left(1+\exp \left(0.083\left(V_{m}+75\right)\right)\right.}
\end{aligned}
$$

where $F_{\tau}$ is a factor that was used in scaling the (de)activation time constant of HCN channels without altering its voltage dependence and the half-maximal activation voltage, $V_{1 / 2}$, was set at a default value of $-82 \mathrm{mV}$ (Magee, 1998).

Ball-and-stick multicompartmental model. Our two-cylinder model comprised a $500-\mu \mathrm{m}$-long and $2-\mu \mathrm{m}$-wide dendrite attached to a 50 $\mu \mathrm{m}$-long, 50- $\mu \mathrm{m}$-wide soma with an axon of length $200 \mu \mathrm{m}$ and diameter $2 \mu \mathrm{m}$ with an axon initial segment (AIS) of length $20 \mu \mathrm{m}$ and diameter $2 \mu \mathrm{m}$ connecting the axon to the soma. The dendrite was divided into 100 compartments to impose the isopotentiality constraint on each compartment. Across locations, $R_{\mathrm{m}}, R_{\mathrm{a}}$, and $C_{\mathrm{m}}$ were uniformly set to $12 \mathrm{k} \Omega \cdot \mathrm{cm}^{2}, 100 \Omega \cdot \mathrm{cm}$, and $1 \mu \mathrm{F} / \mathrm{cm}^{2}$, respectively. The axon was passive, with $R_{\mathrm{m}}, R_{\mathrm{a}}$, and $C_{\mathrm{m}}$ fixed at $24 \mathrm{k} \Omega \cdot \mathrm{cm}^{2}, 100 \Omega \cdot \mathrm{cm}$, and 0.5 $\mu \mathrm{F} / \mathrm{cm}^{2}$, respectively.

The sodium and KDR conductance values were uniformly fixed at $\bar{g}_{N a}=12 \mathrm{mS} / \mathrm{cm}^{2}, \bar{g}_{K D R}=1 \mathrm{mS} / \mathrm{cm}^{2}$ across the somatodendritic compartments (Magee and Johnston, 1995); for nonspiking dendrites, these channel densities were zero for the dendritic compartments. The AIS had the same electrical properties as the soma, but the sodium channel density was 10 times that of the soma (Fleidervish et al., 2010). An additional gating variable was introduced for modeling the current through somatodendritic sodium channels to incorporate slow recovery of these channels from inactivation (Colbert et al., 1997; Migliore et al., 1999) as follows:

$$
I_{N a}=\bar{g}_{N a} m^{3} h s\left(V_{m}-E_{N a}\right)
$$

where $m$ and $h$ are the activation and inactivation gate, respectively, and $s$ is the slow recovery from inactivation. To incorporate slow recovery from inactivation in dendritic channels, the steady-state value of $s, s_{\infty}$, was set as follows (Migliore et al., 1999):

$$
s_{\infty}=\frac{1+F_{r e c}^{N a} \exp \left(\left(V_{m}+58\right) / 2\right)}{1+\exp \left(\left(V_{m}+58\right) / 2\right)}
$$

where $F_{r e c}^{N a}$ is the recovery factor for sodium channels. If $F_{r e c}^{N a}=1$, then $s_{\infty}=1$, thus making the slow inactivation gate irrelevant. If $F_{r e c}^{N a}=0$, then $s_{\infty}=\left(1+\exp \left(\left(V_{m}+58\right) / 2\right)\right)^{-1}$, a sigmoid that represented the voltage dependence of the slow recovery from inactivation. Therefore, the recovery of sodium channels from inactivation was slower if $F_{r e c}$ assumed lower values (in the range of $0-1$ ).

We performed simulations with several somatodendritic gradients of HCN channel densities on models with spiking and nonspiking dendrites: (1) a constant density of HCN channels solely at the soma, (2) a constant density of HCN channels at the soma and the dendrite, and (3) a somatodendritically increasing gradient of $\mathrm{HCN}$ channels of linear or sigmoidal nature. For the last case, when the HCN-channel density increased monotonically from the soma to the distal-most point on the dendrite, parameters for the sigmoidal gradient (Narayanan and Johnston, 2007) were adjusted to match local and transfer resonance properties across the somatodendritic axis ( $\mathrm{Hu}$ et al., 2009; Vaidya and Johnston, 2013). The linear gradient was designed with the same baseline (somatic location) and maximum (terminal dendritic location) values as the sigmoidal gradient to serve as a control. The specific sigmoidal and linear gradients in $\mathrm{HCN}$ channels were set as follows:

$$
\begin{gathered}
\text { Sigmoidal: } \bar{g}_{h}(x)=g_{h}^{\text {Base }}\left(1+\frac{F_{g h}}{1+(\exp ((330-x) / 75))}\right) \\
\text { Linear: } \bar{g}_{h}(x)=g_{h}^{\text {Base }}\left(1+\left(F_{g h}-1\right) x / L\right)
\end{gathered}
$$

where $g_{h}^{\text {Base }}$ is the baseline density of HCN channels with a default value of $28 \mu \mathrm{S} / \mathrm{cm}^{2}, F_{\text {gh }}(=20)$ is the fold increase in the density of HCN channels, and $L(=500 \mu \mathrm{m})$ is the length of the dendrite.

Morphologically realistic model. A three-dimensional reconstruction of a CA1 pyramidal neuron obtained from NeuroMorpho.org (Pyapali et 
al., 1998; Ascoli et al., 2007) was used in constructing a morphologically realistic model. Several experimental constraints were placed on this reconstruction to match its electrophysiological measurements with experimental values. First, passive properties were set such that the input resistance $\left(R_{\text {in }}\right)$ was almost uniform $(\sim 120 \mathrm{M} \Omega)$ across the somatodendritic trunk compartments by setting $R_{\mathrm{m}}$ and $R_{\mathrm{a}}$ as functions of radial distance of the compartment $(x)$ from the soma (Narayanan and Johnston, 2007) as follows:

$$
\begin{aligned}
& R_{m}=R_{m}^{\text {soma }}+\left(\frac{R_{m}^{\text {end }}-R_{m}^{\text {soma }}}{1+\exp ((400-x) / 50)}\right) \\
& R_{a}=R_{a}^{\text {soma }}+\left(\frac{R_{a}^{\text {end }}-R_{a}^{\text {soma }}}{1+\exp ((210-x) / 50)}\right)
\end{aligned}
$$

where $R_{m}^{\text {soma }}=65 \mathrm{k} \Omega \cdot \mathrm{cm}^{2}$ and $R_{a}^{\text {soma }}=50 \Omega \cdot \mathrm{cm}$ were the values at the soma and $R_{m}^{\text {end }}=6 \mathrm{k} \Omega \cdot \mathrm{cm}^{2}$ and $R_{a}^{\text {end }}=30 \Omega \cdot \mathrm{cm}$ were values at the terminal location of the apical trunk ( $\sim 425 \mu \mathrm{m}$ for this model). Apical oblique dendrites had the same $R_{\mathrm{m}}$ and $R_{\mathrm{a}}$ values as the point of their origin on the apical trunk. The basal and axonal compartments had the same $R_{\mathrm{m}}$ and $R_{\mathrm{a}}$ values as the soma. $C_{\mathrm{m}}$ was uniformly set to $1 \mu \mathrm{F} / \mathrm{cm}^{2}$.

In constraining active properties with experimental findings, we introduced $\mathrm{Na}^{+}$and KDR channels into the model with uniform densities across the dendritic arbor with $\bar{g}_{N a}=15 \mathrm{mS} / \mathrm{cm}^{2}, \bar{g}_{K D R}=0.8 \mathrm{mS} / \mathrm{cm}^{2}$, thereby bestowing spiking capabilities upon dendrites as well (Magee and Johnston, 1995; Gasparini et al., 2004; Gasparini and Magee, 2006; Losonczy and Magee, 2006; Bittner et al., 2012). The density of the axonal $\mathrm{Na}^{+}$channels at the AIS was 10 times than that at the soma (Fleidervish et al., 2010). $F_{r e c}^{\mathrm{Na}}$ (Equation 6) of $\mathrm{Na}^{+}$channels was set at 1 for the soma and basal dendrites and at 0.8 for apical dendrites. The axonal $\mathrm{Na}^{+}$ channel model did not have a slow recovery component. For simulations with HCN channels, a gradient in their density along the somatodendritic axis (Magee, 1998; Lörincz et al., 2002) was set as a sigmoidal function (see Fig. $7 B$ ) of the radial distance from soma, $x$ (Narayanan and Johnston, 2007) as follows:

$$
\bar{g}_{h}(x)=g_{h}^{\text {Base }}\left(1+\frac{F_{g h}}{1+(\exp ((250-x) / 25))}\right)
$$

$g_{h}^{\text {Base }}$ represented the somatic density of HCN channels, with a default value of $45 \mu \mathrm{S} / \mathrm{cm}^{2}$, and $F_{g \mathrm{~h}}(=20)$ quantified the fold increase in the density of HCN channels. The half-maximal voltage for activation of $\mathrm{HCN}$ channels was varied along the somatodendritic axis such that it was $-82 \mathrm{mV}$ for $x \leq 100 \mu \mathrm{m}$, increased linearly from $-82 \mathrm{mV}$ to $-90 \mathrm{mV}$ for $100>x \geq 300 \mu \mathrm{m}$, and $-90 \mathrm{mV}$ for $x>300 \mu \mathrm{m}$ (Magee, 1998). Incorporation of $\mathrm{HCN}$ channels with these gradients resulted in reducing the $R_{\text {in }}$ from $\sim 50 \mathrm{M} \Omega$ at the soma to $\sim 29 \mathrm{M} \Omega$ distally. The local resonance frequency $\left(f_{\mathrm{R}}\right)$ increased from 5 to $9 \mathrm{~Hz}$, whereas the transfer resonance frequency $\left(f_{\mathrm{TR}}\right)$ varied from $\sim 5$ to $7 \mathrm{~Hz}$ (see Fig. $7 B-D$ ). The $\mathrm{HCN}$ channel gradient parameters were tuned to match all three of these measurements $\left(R_{\mathrm{in}}, f_{\mathrm{R}}\right.$ and $\left.f_{\mathrm{TR}}\right)$ with corresponding experimental findings across the somatoapical trunk (Magee, 1998; Lörincz et al., 2002; Narayanan and Johnston, 2007; Hu et al., 2009; Vaidya and Johnston, 2013).

\section{Physiological measurements}

The input resistance, $R_{\text {in }}$, of a neuronal compartment was computed by measuring the steady-state voltage response to current pulses of -50 to $50 \mathrm{pA}$ in steps of $10 \mathrm{pA}$ for $500 \mathrm{~ms}$ each. The steady-state voltage response was then plotted against the current amplitude and the slope of this linear relationship constituted $R_{\text {in }}$ of that compartment. The local resonance frequency, $f_{\mathrm{R}}$, of a neuronal compartment was computed by injecting a chirp stimulus spanning $25 \mathrm{~Hz}$ in $25 \mathrm{~s}$. The local voltage response was recorded and its Fourier transform magnitude divided by the Fourier transform magnitude of the injected chirp stimulus was defined as the local impedance amplitude profile (ZAP) of the compartment. The frequency at which the ZAP reached its maximum $\left(\left|Z_{\text {local }}\right|_{\max }\right)$ was designated as $f_{\mathrm{R}}$ of the neuronal compartment (Narayanan and Johnston, 2007). A similar procedure was adopted in computing the transfer resonance frequency $\left(f_{\mathrm{TR}}\right)$, with the only difference being that the voltage response was measured at the soma, rather than at the location of current injection. We denoted the maximum value of the transfer impedance amplitude profile (across the measured frequencies) as $\left|Z_{\mathrm{TR}}\right|_{\max }$, which by definition would be equal to $\left|Z_{\mathrm{TR}}\left(f_{\mathrm{TR}}\right)\right|$, and used it to measure transfer resonance strength $\left(Q_{\mathrm{TR}}\right)$ as the ratio of $\left|Z_{\mathrm{TR}}\left(f_{\mathrm{TR}}\right)\right|$ to $\left|Z_{\mathrm{TR}}(0.5)\right|$. The fast after-hyperpolarization potential (fAHP) was measured as the absolute difference between the resting membrane potential and the most hyperpolarized voltage value in a $10 \mathrm{~ms}$ window that followed the peak of an action potential. Suprathreshold resonance frequency $\left(f_{\mathrm{SR}}\right)$ was computed by injecting noisy (Gaussian white) sinusoidal current of different frequencies $(1-15 \mathrm{~Hz})$ to the somatic or dendritic compartments and measuring the somatic firing rate (Fig. $1 H$ ). The frequency of the input sinusoid for which the cell elicited the maximal firing response was measured as the $f_{\mathrm{SR}}$ for the input location where the current was injected.

\section{Computing spike-triggered average}

To estimate the STA, zero-mean Gaussian white noise (GWN) with an $\mathrm{SD} \sigma_{\text {noise }}$ was injected into a neuronal compartment. The mean was set to zero and $\sigma_{\text {noise }}$ was adjusted to a minimum value required for firing action potentials at an average of $\sim 1 \mathrm{~Hz}$ for a given parametric variation. This ensured that the spikes remained isolated and aperiodic (Fig. 1A), thereby establishing statistical independence of the samples used for arriving at the STA (Famulare and Fairhall, 2010). In performing sensitivity analysis with reference to any given parameter, the range of the concerned parameter was fixed such that this constraint on isolated spikes was accounted for. The STA was calculated for a period of 300 $\mathrm{ms}$ preceding the spike and averaged over all recorded spikes across the time period of the simulation. For multicompartmental models, the GWN current with appropriate $\sigma_{\text {noise }}$ was injected at different locations along the dendrite and the spikes were recorded at the soma to compute the dendritic STA. STA kernels were smoothed for representational purposes and for computing measurements that we derived from the STA. Across models and parametric variations, the total time of simulation was adjusted such that no less than 1000 spikes were harvested toward computing the STA. Given the low firing frequency of $\sim 1 \mathrm{~Hz}$, this meant a simulation time period of $\sim 1000$ s for computing a single STA.

\section{Spectrotemporal representation of the STA and computational details}

To assess the temporal localization of spectral selectivity in spike initiation dynamics, wavelet analysis was performed on the STA using the complex Morlet wavelet (Grossman and Morlet, 1984; Torrence and Compo, 1998; Le Van Quyen and Bragin, 2007). The continuous wavelet transform of a signal $x(t)$ was defined as its convolution with a wavelet function as follows:

$$
W_{x}(\tau, s)=\int_{-\infty}^{\infty} \frac{x(t)}{\sqrt{s}} \psi^{\star}\left(\frac{t-\tau}{s}\right) d t
$$

where $\psi^{*}(t)$ is the complex conjugate of the wavelet function $\psi(t)$, s is the dilation parameter, and $\tau$ is the translation parameter of the wavelet. The complex Morlet wavelet function $\psi(t)$ was defined as follows:

$$
\psi(t)=\pi^{-1 / 4} \exp \left(\frac{-t^{2}}{\sigma_{b}}+j \omega_{c} t\right)
$$

where $\sigma_{\mathrm{b}}(=2 \mathrm{~Hz}$ for the mother wavelet $)$ is the bandwidth parameter and $\omega_{\mathrm{c}}(=5 \mathrm{~Hz}$ for the mother wavelet $)$ is the center frequency of the wavelet. Wavelet coefficients (Equation 13) were computed on the STA (over $300 \mathrm{~ms}$ before a spike) for 800 scales, starting at $s=0.01$ with increments of $s$ at 0.001 . This allowed us to compute wavelet coefficients spanning the frequency range of $1-85 \mathrm{~Hz}$. To delineate temporal localization of feature selectivity and changes in the integration time window of the STA kernel, we converted scales to frequencies and plotted the amplitude of wavelet coefficients for various values of $s$ on a timefrequency plane represented on linear axes (Fig. 1E).

The NEURON simulation environment (Carnevale and Hines, 2006) was used for modeling and all analyses were performed using custom- 
A

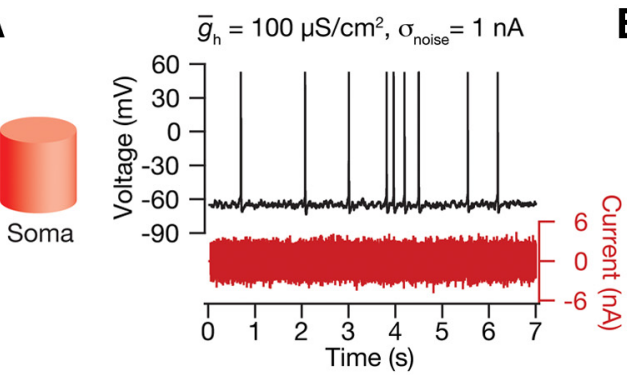

D

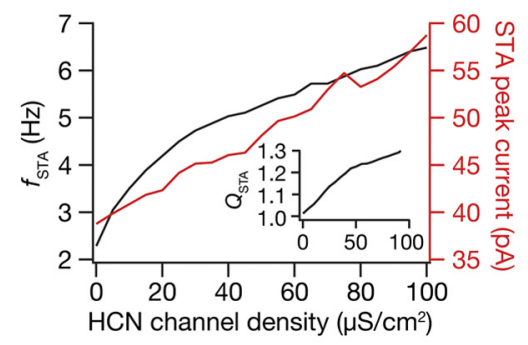

B

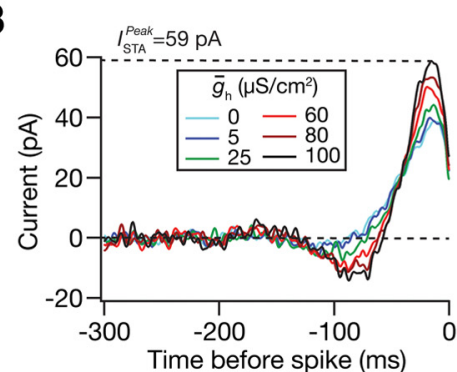

E

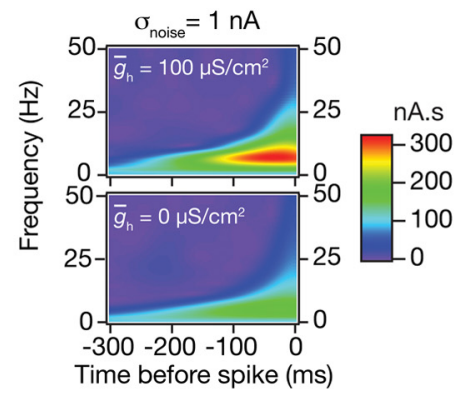

C

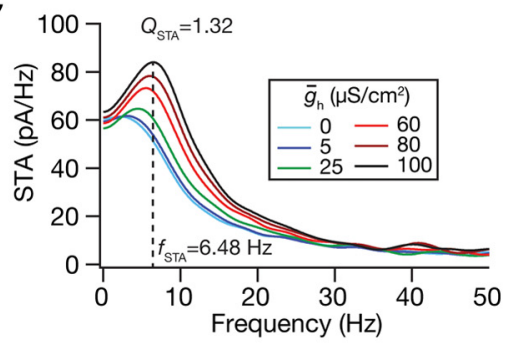

F

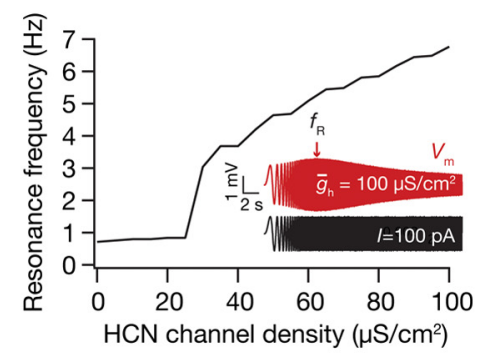

G

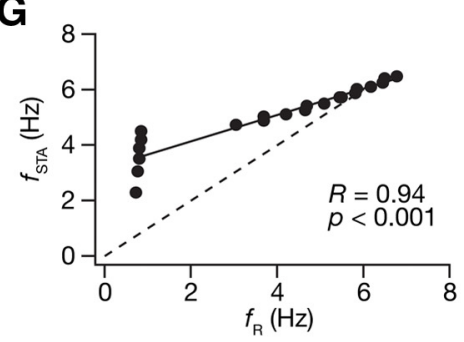

H

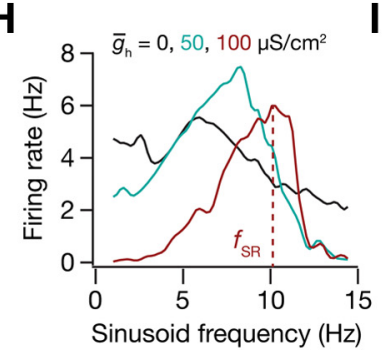

I

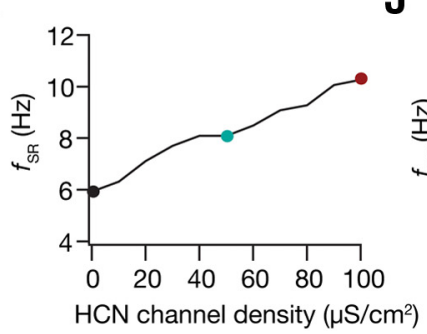

J

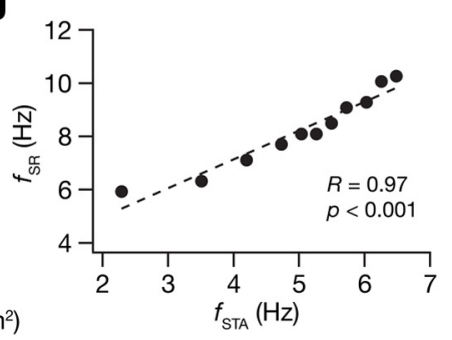

Figure 1. STA exhibits theta-frequency selectivity in the presence of HCN channels. A, Left, Depiction of single-compartmental model used for all results presented in this figure. Right, Injection of zero mean Gaussian white noise (red) elicited action potentials in the single compartmental model neuron (voltage trace in black). $\boldsymbol{B}$, The STA in the presence of HCN channels resembled a damped oscillatory waveform, with a direct relationship between the oscillatory amplitude and the density of HCN channels. C, The Fourier transform of the STA revealed its band-pass nature and facilitated the quantification of frequency selectivity (at $\left.f_{S T A}\right)$ and selectivity strength $\left(Q_{S T A}\right) \cdot \boldsymbol{D}, I_{S T A}^{\text {Peak }}, f_{S T A}$, and $Q_{S T A}$ increased with increasing density of HCN channels. $E$, Wavelet analysis of the STA revealed spectrotemporally localized theta-frequency selectivity in the presence of $\mathrm{HCN}$ channels (top), which was absent in the absence of $\mathrm{HCN}$ channels (bottom). $\boldsymbol{F}$, Subthreshold resonance frequency ( $f_{\mathrm{R}}$ ) increased with increasing HCN-channel density. Inset, Chirp current and voltage response of a model with HCN channel density set at $100 \mu \mathrm{S} / \mathrm{cm}^{2}$. G, $f_{\text {STA }}$ and $f_{\mathrm{R}^{\prime}}$ computed at different values of HCN-channel density exhibited a significantly strong correlation (Pearson's correlation test). $\boldsymbol{H}, \boldsymbol{I}$, Injection of noisy sinusoidal input into the same model neuron resulted in a sinusoidal frequencydependent firing rate $(\boldsymbol{H})$, with the suprathreshold resonance frequency $\left(f_{\mathrm{SR}}\right)$ increasing with increases in $\mathrm{HCN}$ channel density $(\boldsymbol{I})$. $\boldsymbol{J}, f_{\mathrm{STA}}$ and $f_{\mathrm{SR}}$, computed in models expressing $\mathrm{HCN}$ channels at different densities, exhibited a significantly strong correlation (Pearson's correlation test).

built software written in Igor Pro (Wave Metrics). All simulations were done at $34^{\circ} \mathrm{C}$ with an integration time step of $25 \mu$ s. Statistical analyses were done using the R statistical package (http://www.r-project.org).

\section{List of symbols and abbreviations}

The symbols and abbreviations used in the manuscript are: AIS, axon initial segment; $C_{\mathrm{m}}$, specific membrane capacitance; $E_{\mathrm{h}}$, the HCNchannel reversal potential; fAHP, fast after-hyperpolarization potential; $f_{\mathrm{R}}$, local resonance frequency; $f_{\mathrm{SR}}$, suprathreshold resonance frequency; $f_{\mathrm{STA}}$, STA characteristic frequency; $f_{\mathrm{TR}}$, transfer resonance frequency; $F_{r e c}^{N a}$, factor governing recovery of sodium channels from inactivation; $\bar{g}_{h}$, maximal HCN conductance; $g_{n}^{\text {Base }}$, baseline (somatic) density of HCN channels; $\bar{g}_{K D R}$, delayed rectifier potassium channel density; $\bar{g}_{N A}$, sodium channel density; GWN, Gaussian white noise; HCN, hyperpolarizationactivated cyclic nucleotide-gated channels; I-CD continuum, integrator-coincidence detector continuum; $I_{S T A}^{\text {Peak }}$, positive peak of the STA; $\mathrm{KDR}$, delayed rectifier potassium; MISO system, multi-input-singleoutput system; $Q_{R}$, local resonance strength; $Q_{\mathrm{STA}}$, STA selectivity strength; $Q_{\mathrm{TR}}$, transfer resonance strength; $R_{\mathrm{a}}$, specific intracellular resistivity; $R_{\text {in }}$, input resistance; $R_{\mathrm{m}}$, specific membrane resistivity; $\sigma_{\text {noise }}, \mathrm{SD}$ of Gaussian white noise; STA, spike-triggered average; $\tau_{\mathrm{HCN}}$, time constant of HCN channels; VGIC, voltage-gated ion channels; $V_{\mathrm{m}}$, the membrane voltage; ZAP, impedance amplitude profile; $\left|Z_{\text {local }}\right|_{\text {max }}$, maximal amplitude of local impedance profile; $\left|Z_{\mathrm{TR}}\right|_{\max }$, maximal amplitude of transfer impedance profile.

\section{Results}

The STA, also known as the first-order Wiener kernel (Rieke et al., 1999), is a well established measure for understanding neural coding of input features and has been used effectively to unravel the selectivity of single-neuronal spikes to specific features that are inherent to afferent synaptic inputs. Whereas a single STA kernel is used in the linear characterization of a point neuron, it is not known if the STA is input-invariant across the span of a pyramidal neuron endowed with plastic active dendrites. To address this, we first developed several physiologically relevant quantitative measurements from the temporal and spectral representations of the STA and then used these measurements to assess the roles of the kinetics and voltage dependence of several dendritically expressed VGICs on spike initiation dynamics. Apart from spike-related VGICs (fast $\mathrm{Na}^{+}$and KDR), we chose $\mathrm{HCN}$ channels as a critical example for our analyses for multiple reasons (Magee, 1998, 2000; Lörincz et al., 2002; Ulrich, 2002; Narayanan and Johnston, 2007, 2008; Hu et al., 2009; Vaidya and 
Johnston, 2013). First, HCN channels are active at subthreshold voltages and play a critical role in neuronal integration before spike initiation. Second, $\mathrm{HCN}$ channels are critical regulators of subthreshold frequency selectivity and form an ideal substrate with which to assess the relationship between subthreshold resonance and spike initiation dynamics. Third, HCN channels display a predominant dendritic expression profile, enabling our analyses of how dendritic VGICs regulate spike initiation dynamics. Finally, experimental data about HCN channel properties (voltage dependence, kinetics, and distribution across the somatodendritic arbor), along with physiological properties critically dependent on the HCN channels (input resistance, local and transfer resonance frequency along the somatodendritic arbor), were available from CA1 pyramidal neurons, allowing us to constrain the expression profile and the consequent physiological properties in our model.

STA characteristic frequency $\left(f_{\mathrm{STA}}\right)$ and selectivity strength $\left(Q_{\text {STA }}\right)$ as measures of frequency selectivity in spike initiation dynamics

HCN channels are well established mediators of subthreshold theta frequency $(4-10 \mathrm{~Hz})$ selectivity in hippocampal pyramidal neurons (Narayanan and Johnston, 2007; Hu et al., 2009). Does subthreshold resonance in the theta frequency range translate to theta-frequency selectivity in spike initiation dynamics? To answer this question, we used a minimal single compartmental model of a neuron that expressed sodium and KDR channels for spike initiation and measured STA with various conductance densities of HCN channels. In the absence of $\mathrm{HCN}$ channels, the STA closely resembled that of class 1 (C1) excitability (Hodgkin, 1948) neurons that displayed integrator-like traits (Prescott et al., 2008a; Prescott et al., 2008b; Hong et al., 2012; Ratté et al., 2013). Specifically, STA remained largely positive, with larger current values closer to the spike, and progressively declined with temporal distance from the spike initiation point (Fig. 1B). With the insertion of $\mathrm{HCN}$ channels, we found that a damped oscillatory pattern characterized the STA, with traits similar to that of class $2 / 3$ (C2/C3) neurons that tend to act as coincidence detectors (Badel et al., 2006; Prescott et al., 2008a; Hong et al., 2012; Ratté et al., 2013). Therefore, an increase in HCN channel density $\left(\bar{g}_{h}\right)$ induced a progressive shift from C1-like STAs to C3-like STAs through C2-like STAs, thereby effectuating a physiological transition from an integrator to a coincidence detector.

What characteristics of an STA could be used as quantitative, physiologically relevant measures of spike initiation dynamics and would best describe its progressive transition from an integrator to a coincidence detector? First, the positive peak of the STA $\left(I_{S T A}^{\text {Peak }}\right)$ represents the amount of current required for eliciting a spike, thereby lending itself as a direct measure of neuronal excitability (Fig. 1B). Second, although the low-pass nature of C1 neurons' STA and the band-pass nature of $\mathrm{C} 2 / \mathrm{C} 3$ neurons' STA are established (Prescott et al., 2008a; Hong et al., 2012; Ratté et al., 2013), this frequency selectivity in single neuron spiking $d y-$ namics has not been characterized quantitatively. We quantified this frequency selectivity by transforming the STA to the spectral domain using the Fourier transform and defined the frequency where the Fourier transform magnitude of the STA $(|\operatorname{STA}(f)|)$ peaked as the STA characteristic frequency $\left(f_{\text {STA }}\right)$. Under this formulation, $f_{\mathrm{STA}} \approx 0$ would imply a low-pass STA $(\mathrm{C} 1)$ and a nonzero $f_{\text {STA }}$ would refer to a band-pass structure (C2/C3) peaking at $f_{\mathrm{STA}}$. Third, we defined STA selectivity strength $\left(Q_{\mathrm{STA}}\right)$ as the ratio of $\left|\operatorname{STA}\left(f_{\mathrm{STA}}\right)\right|$ to $|\operatorname{STA}(0.5 \mathrm{~Hz})|$; a larger $Q_{\mathrm{STA}}$ indicated stronger feature selectivity, whereas $Q_{\mathrm{STA}}$ values closer to unity reflected weak selectivity.

\section{STA characteristic frequency and selectivity strength increased monotonically with increases in HCN channel density}

We used these three measures to assess quantitatively the role of HCN channels in defining spike initiation dynamics. We noted that $I_{S T A}^{\text {Peak }}$ increased with increasing $\bar{g}_{h}$ (Fig. $1 B, D$ ), reflecting the reduction in excitability of the neuron brought about by the increased conductance of these channels (Magee, 1998, 2000; Fan et al., 2005; Narayanan and Johnston, 2007). Increases in $\bar{g}_{h}$ also resulted in a monotonic increase in the $f_{\mathrm{STA}}$ and $Q_{\mathrm{STA}}$ (Fig. $1 D$ ). Importantly, $f_{\mathrm{STA}}$ did not approach zero ( $f_{\mathrm{STA}}$ approaching zero implies an absolutely low-pass STA structure) even when no HCN channels were present, suggesting that the STA band-pass structure was not exclusively dependent on the presence of resonating conductances (see Fig. 3).

Although the Fourier transform yielded a spectral representation of the STA, it does not evince information about the specific temporal locations (with reference to the spike) where the spectral selectivity expresses maximally. To understand the spectrotemporal structure of spike initiation dynamics, we performed wavelet analysis of STA computed in the presence and absence of the HCN channels. We found that the presence of $\mathrm{HCN}$ channels endowed the neuron with theta-frequency selectivity and the spectral selectivity was temporally localized to $\sim 100 \mathrm{~ms}$ before spike initiation (Fig. 1E). Conversely, the absence of HCN channels abolished theta frequency selectivity, yielding a typical lowpass response characteristic associated with integrators (Fig. 1E).

\section{STA characteristic frequency of the neuron is positively correlated with its subthreshold and suprathreshold resonance frequency}

Is there a relationship between the STA characteristic frequency and the subthreshold resonance frequency of the compartment? We plotted $f_{\text {STA }}$ (Fig. $1 D$ ) against $f_{\mathrm{R}}$ (Fig. $1 F$ ) for various values of $\bar{g}_{h}$, with both measures computed from models with identical active and passive properties. We found that $f_{\text {STA }}$ was strongly correlated with $f_{R}$, emphasizing the critical role of a subthreshold resonating conductance in determining spectral selectivity in spike initiation dynamics (Fig. 1G). Does spectral selectivity in the STA kernel translate to a frequency preference in the firing rate of the neuron responding to suprathreshold oscillatory inputs? To answer this question, we injected noisy suprathreshold sinusoidal currents of the same amplitude but different frequencies $(1-15 \mathrm{~Hz})$ into the neuron and measured the firing rate for each input frequency (Fig. $1 H$ ). The firing rate was dependent on the input sinusoidal frequency and exhibited a preferred input frequency for maximal firing. We quantified the preferred input frequency as the suprathreshold resonance frequency $\left(f_{\mathrm{SR}}\right)$ and found that $f_{\mathrm{SR}}$ increased with increase in HCN channel density (Fig. $1 I$ ). Importantly, $f_{\mathrm{STA}}$ was strongly correlated with $f_{\mathrm{SR}}$ (Fig. $1 J$ ), verifying the relationship between the kernel-based $f_{\mathrm{STA}}$ and firing-response-based $f_{\mathrm{SR}}$.

\section{Spectral selectivity in spike initiation dynamics is critically regulated by stimulus statistics and by the kinetics and voltage dependence of $\mathrm{HCN}$ channels}

The dependence of the STA on noise variance (or, equivalently, on stimulus contrast) is well established (Chander and Chichilnisky, 2001; Yu and Lee, 2003; Hong et al., 2007; Famulare and 
A

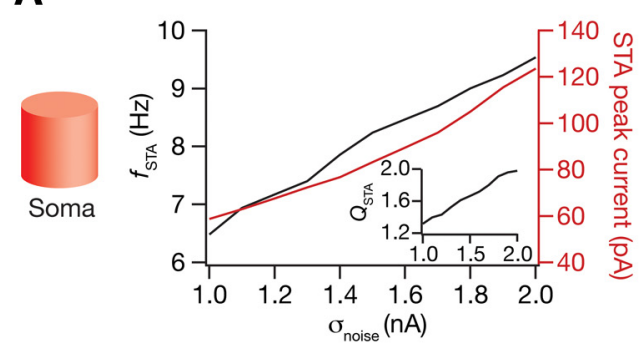

D

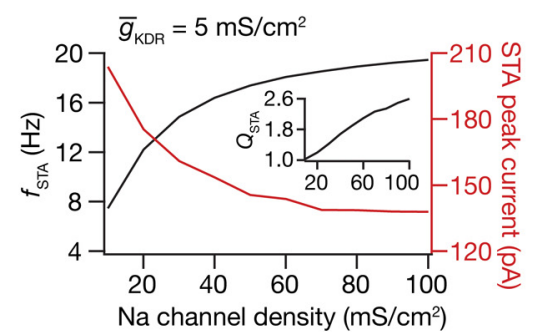

B

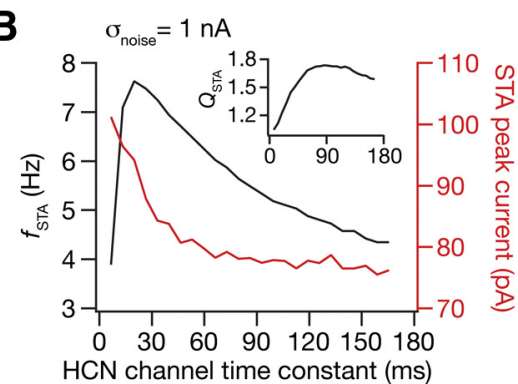

C

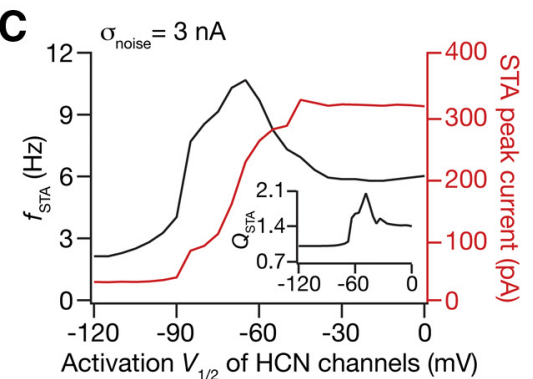

E

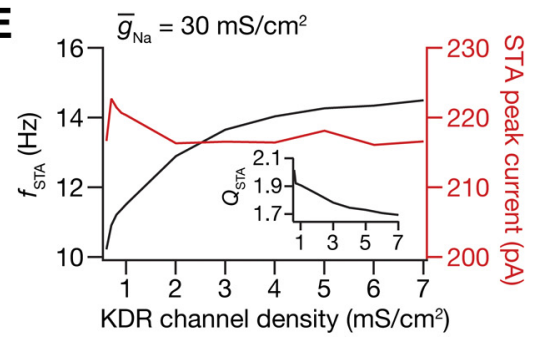

$\mathbf{F}$

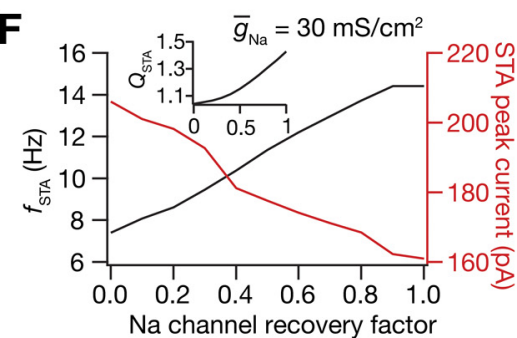

Figure 2. STA characteristic frequency was dependent on input strength and on properties of voltage-gated ion channels. $A$, Left, Depiction of single-compartmental model used for all results presented in this figure. $\boldsymbol{A}-\boldsymbol{F}$, STA characteristic frequency $\left(f_{\mathrm{STA}}\right)$, the peak STA current $\left(I_{S T A}^{\text {Peak }}\right)$, and STA selectivity strength $\left(Q_{S T A}\right.$; insets) plotted as functions of the SD of the Gaussian white noise, $\sigma_{\text {noise }}(\boldsymbol{A})$, (de)activation time constant of HCN channels, $\tau_{\mathrm{HCN}}(\boldsymbol{B})$, activation $V_{1 / 2}$ of HCN channels $(\boldsymbol{C})$, maximal sodium channel density, $\bar{g}_{N a}(\boldsymbol{D})$, maximal KDR channel density, $\bar{g}_{K D R}(\boldsymbol{E})$, and the factor governing the recovery of sodium channels from inactivation (Equation 6), $\boldsymbol{F}_{r e c}^{N a}(\boldsymbol{F})$. For $\boldsymbol{A}-\boldsymbol{F}, \bar{g}_{h}=100 \mu \mathrm{S} / \mathrm{cm}^{2} ;$ for $\boldsymbol{A}-\boldsymbol{C}, \bar{g}_{K D R}=0.6 \mathrm{mS} / \mathrm{cm}^{2}$ and $\bar{g}_{\mathrm{Na}}=30 \mathrm{mS} / \mathrm{cm}^{2}$; for $\boldsymbol{D}-\boldsymbol{F}, \sigma_{\text {noise }}=1 \mathrm{nA}$.

Table 1. Sensitivity analysis for STA measurements corresponding to various passive and active parameters in the single compartmental model

\begin{tabular}{|c|c|c|c|c|c|}
\hline & Increase in & Tested range & $I_{S T A}^{\text {Peak }}$ & $f_{\text {STA }}$ & $Q_{\text {STA }}$ \\
\hline \multirow[t]{3}{*}{ Passive } & $C_{m}$ & $1-1.5$ & Increases & Decreases & Decreases \\
\hline & $R_{\mathrm{m}}$ & $20-40$ & Decreases & Increases & Increases \\
\hline & $V_{\mathrm{m}}$ & -69 to -59 & Decreases & Bell-shaped & Bell-shaped \\
\hline \multirow[t]{3}{*}{ HCN channel } & $\bar{g}_{N a}^{m}$ & $0-100$ & Increases & Increases & Increases \\
\hline & $V_{1 / 2}$ act & $-120-0$ & Increases & Bell-shaped & Bell-shaped \\
\hline & $\tau_{\mathrm{HCN}}$ & $3-165$ & Decreases & Decreases after a threshold & Asymmetric bell-shaped \\
\hline \multirow[t]{5}{*}{ Fast sodium channel } & $\bar{g}_{\mathrm{Na}}$ & $10-100$ & Decreases & Increases & Increases \\
\hline & $V_{1 / 2}$ act & -32 to -28 & Increases & Decreases & Decreases \\
\hline & $V_{1 / 2}$ inact & -50 to -35 & U-shaped ${ }^{a}$ & Increases $^{a}$ & Decreases $^{a}$ \\
\hline & $\tau_{\text {inact }}$ & $1-22$ & Decreases & Increases & Decreases \\
\hline & $I_{S T A}^{P \text { Pack }}$ & $0-1$ & Decreases & Increases & Increases \\
\hline \multirow[t]{3}{*}{ Delayed Rectifier potassium channel } & $\bar{g}_{K D R}$ & $0.6-7$ & Increases & Increases & Decreases \\
\hline & $V_{1 / 2}$ act & -15 to -5 & Increases $^{a}$ & Decreases & Increases \\
\hline & $\tau_{\mathrm{act}}$ & $2-5$ & Increases $^{a}$ & Increases $^{a}$ & Decreases $^{a}$ \\
\hline
\end{tabular}

Shown are changes in the STA peak current, STA characteristic frequency, and the STA selectivity strength obtained from simulations in which each of the multicompartmental parametric values was increased. Increases in the voltages $\left(V_{m}\right.$ and $\left.V_{1 / 2}\right)$ indicate shifts toward more depolarized potentials. An increase in $V_{1 / 2}$ activation or inactivation corresponds to a depolarizing shift. Units are as follows: $C_{\mathrm{m}}, \mu \mathrm{F} / \mathrm{cm}^{2} ; R_{\mathrm{m}}, \mathrm{k} \Omega . \mathrm{cm}^{2} ; V_{\mathrm{m}}, \mathrm{mV} ; \bar{g}_{h}, \mu S / \mathrm{cm}^{2} ;$ all voltages, $\mathrm{mV} ; \tau_{\mathrm{HCN}}$, $\mathrm{ms} ; \bar{g}_{\mathrm{Na},} \mathrm{mS} / \mathrm{cm}^{2} ; \tau_{\text {inact }}, \mathrm{ms} ; \bar{g}_{K D R}, \mathrm{mS} / \mathrm{cm}^{2} ; \tau_{\text {act }}, \mathrm{ms}$.

${ }^{a}$ Small changes.

Fairhall, 2010). To understand gain control in our CA1 pyramidal neuronal model in response to stimulus variance, we computed STA when the neuronal model was presented with GWN of different values for $\sigma_{\text {noise }}$. We found that increases in stimulus variance altered spike initiation dynamics by modulating its spectral selectivity through an increase in $f_{\mathrm{STA}}$, apart from increasing $I_{S T A}^{P e a k}$, and increasing $Q_{\mathrm{STA}}$ (Yu and Lee, 2003; Famulare and Fairhall, 2010; Fig. 2A).

The dependence of STA measurements on input statistics and its bell-shaped dependence on $V_{\mathrm{m}}$ (Table 1) suggested an obvious role for voltage-dependent channel properties in regulating spike initiation dynamics (Hong et al., 2007; Prescott et al., 2008a; Hong et al., 2012; Ratté et al., 2013). The slow activation time constant of $\mathrm{HCN}$ channels $\left(\tau_{\mathrm{HCN}}\right)$ forms a critical requirement in its ability to act as a resonating conductance; resonance is not sustained by a faster HCN channel (Hutcheon et al., 1996; Naray- anan and Johnston, 2007, 2008). Altering $\tau_{\mathrm{HCN}}$ in our model, we found that $f_{\mathrm{STA}}$ followed a profile that was similar to that of the dependence of $f_{\mathrm{R}}$ on $\tau_{\mathrm{HCN}}$ (Narayanan and Johnston, 2008), whereby after a threshold value of $\tau_{\mathrm{HCN}}, f_{\mathrm{STA}}$ jumped to a higher frequency value and progressively declined with further increases in $\tau_{\mathrm{HCN}}$ (Fig. $2 B$ ). In addition, increases in $\tau_{\mathrm{HCN}}$ monotonically reduced $I_{S T A}^{\text {Peak }}$, a direct reflection of the increase in impedance amplitude (and therefore excitability) after increases in $\tau_{\mathrm{HCN}}$ (Narayanan and Johnston, 2008). $Q_{\text {STA }}$ displayed an asymmetric bell-shaped dependence on $\tau_{\mathrm{HCN}}$ (Fig. $2 B$ ), again reflective of how subthreshold resonance strength, $Q_{\mathrm{R}}$, depended on $\tau_{\mathrm{HCN}}$ (Narayanan and Johnston, 2008).

The impact of voltage dependence properties of $\mathrm{HCN}$ channels on STA measurements also resembled their impact on subthreshold resonance properties. Specifically, $f_{\mathrm{STA}}$ and $Q_{\mathrm{STA}}$ displayed asymmetric bell-shaped dependencies on the half- 
maximal activation voltage ( $\left.V_{1 / 2}\right)$ of HCN channels (Fig. $2 C$ ), reflective of the bell-shaped dependence of $f_{\mathrm{R}}$ and $Q_{\mathrm{R}}$ on the activation $V_{1 / 2}$ of HCN channels, respectively (Narayanan and Johnston, 2008). $I_{S T A}^{\text {Peak }}$ manifested a sigmoidal increase when we introduced depolarizing shifts in the activation $V_{1 / 2}$ (Fig. 2C), a reflection of the sigmoidal reduction in the impedance amplitude with depolarization in the HCN-channel activation $V_{1 / 2}$ (Narayanan and Johnston, 2008). Therefore, STA measurements were critically dependent upon $\mathrm{HCN}$-channel kinetics and voltage dependence, with the dependencies of $f_{\text {STA }}, Q_{\text {STA }}$, and $I_{S T A}^{\text {Peak }}$ reflective of respective dependencies of $f_{\mathrm{R}}, Q_{R}$, and maximal impedance amplitude. All STA measurements were also modulated by the resting and passive properties of the neuron (Table 1) and largely mirrored the dependence of corresponding subthreshold impedance measurements on respective parameters (Narayanan and Johnston, 2008).

\section{Spike-related active conductances govern the convergence of the STA characteristic frequency to subthreshold resonance frequency in the presence of $\mathrm{HCN}$ channels}

Next, we performed systematic sensitivity analyses involving the parameters associated with the fast $\mathrm{Na}^{+}$and $\mathrm{KDR}$ conductances in the presence of HCN channels (Fig. 2, Table 1). Surprisingly, we found that $f_{\mathrm{STA}}$ increased in response to increases in either the $\mathrm{Na}^{+}\left(\bar{g}_{N a}\right.$; Fig. $2 D$, Table 1$)$ or the KDR ( $\bar{g}_{K D R}$; Fig. $2 E$, Table 1$)$ channel densities, parameters that we found to not significantly alter $f_{\mathrm{R}}$. In contrast, whereas an increase in the $\bar{g}_{\mathrm{Na}}$ increased $Q_{\mathrm{STA}}$ and decreased $I_{S T A}^{\text {Peak }}$ (Fig. $2 D$, Table 1), an increase in $\bar{g}_{K D R}$ decreased $Q_{\text {STA }}$ and did not significantly alter $I_{S T A}^{\text {Peak }}$ (Fig. 2E, Table 1). Importantly, we found that $f_{\mathrm{STA}}$ approached $f_{\mathrm{R}}$ when $\bar{g}_{\mathrm{Na}}$ or $\bar{g}_{K D R}$ were brought down to lower values. The kinetics and voltagedependence properties of the spiking-related channels also had a significant impact on STA measurements (Table 1). Specifically, in the presence of sodium channels that recovered slowly from inactivation (by varying $F_{r e c}^{\mathrm{Na}}$ in Equation 6), $f_{\mathrm{STA}}$ reduced and converged to $f_{\mathrm{R}}$, even in the presence of higher values of $\bar{g}_{\mathrm{Na}}$ and $\bar{g}_{K D R}$ (cf. Fig. $2 F, D$ ). In summary, spike-related conductances critically modulate the spike initiation dynamics and dendritic sodium channels in hippocampal pyramidal neurons that exhibit slower recovery from inactivation (Colbert et al., 1997; Migliore et al., 1999) could play a critical role in regulating the equivalence of subthreshold resonance and STA spectral selectivity.

\section{Spike-related active conductances govern weak spectral selectivity in the spike initiation dynamics in the absence of HCN channels}

How do spike-related conductances alter STA measurements in the absence of subthreshold resonance introduced by HCN channels? In the absence of HCN channels, the model exhibited weak (low values of $Q_{\text {STA }}$ ) delta frequency $(0.5-4 \mathrm{~Hz})$ selectivity in the STA $\left(\bar{g}_{h}=0\right.$; cf. Fig. $\left.1 B-D\right)$. Increasing either $\bar{g}_{N a}$ or $\bar{g}_{K D R}$ resulted in higher values of $f_{\mathrm{STA}}$ and the introduction of $\mathrm{Na}^{+}$channels that recovered slowly from inactivation eliminated the band-pass characteristics of the spike initiation dynamics (Table 1). What mediated this weak STA spectral selectivity? First, we confirmed that neither the $\mathrm{Na}^{+}$nor the KDR channels were capable of sustaining resonance at subthreshold or near-threshold voltages, suggesting that frequency selectivity in STA was independent of subthreshold resonance. After detailed sensitivity analyses involving different parameters associated with the kinetics and voltage dependence of the $\mathrm{Na}^{+}$and the KDR channels, we arrived at a strong correlation between $f_{\mathrm{STA}}$ and the spike fAHP across parametric variations (Fig. $3 A-C$ ). Specifically, examination of the voltage traces of the neuron revealed an increased fAHP with increased $\bar{g}_{N a}$ (Fig. 3A), $\bar{g}_{K D R}$ (Fig. 3B), or $F_{r e c}^{\mathrm{Na}}$ (Fig. 3C) and fAHP computed in all cases strongly and significantly correlated with corresponding values of $f_{\text {STA }}$ (Fig. $3 A-C$ ). In summary, our results thus far (Figs. 1, 2, 3) suggested that any biophysical mechanism that enhances the spiking probability of the neuron after a hyperpolarization of the membrane potential results in a bandpass structure (nonzero $f_{\mathrm{STA}}$ ) for the STA in the spectral domain.

\section{Neuronal model with a spiking soma and a passive dendrite exhibits weak spectral selectivity in spike initiation dynamics in the absence of HCN channels}

The STA forms a first-order kernel that approximates the inputoutput relationship of a neuron. Although studies involving the analyses of STA typically confine themselves to the neuronal soma, the majority of synaptic inputs onto pyramidal neurons arrive at their dendrites (Megías et al., 2001; Spruston, 2008). How does the presence of dendrites alter the spike initiation dynamics of a neuron? How do cable filtering and the presence and plasticity of various dendritic voltage-gated ion channels (Rall, 1977; Magee, 2000; Williams and Stuart, 2003; Lai and Jan, 2006; Johnston and Narayanan, 2008; Sjöström et al., 2008; Spruston, 2008; Shah et al., 2010; Narayanan and Johnston, 2012; Nusser, 2012) alter frequency selectivity in the spike initiation dynamics of a neuron? To answer these questions, we first computed STA using somatic spike timings recorded from a ball-and-stick model with a spiking soma ( $\mathrm{Na}^{+}$and KDR channels inserted) and a passive dendrite. We injected the GWN current at different locations along the somatodendritic axis to assess spike initiation dynamics for inputs arriving at those specific locations. Consistent with our results with a single-compartmental model lacking HCN channels (Fig. 3A-C), the ball-and-stick model manifested weak frequency selectivity at delta frequencies (Fig. $3 G$ ) in the absence of HCN channels. As the location of current became distal to the soma, we observed that: (1) stronger distal current inputs were required for eliciting a somatic action potential (Fig. $3 D, E)$, reflecting as an increase in $I_{S T A}^{\text {Peak }}$ with increasing dendritic distance from the soma (Fig. 3G); (2) the temporal location of the peak of the STA shifted away from the spike time with increasing distance (Fig. 3D); and (3) the STA was broader for distal inputs compared with that for proximal inputs (Fig. $3 D$ ), suggesting that the integration time window was higher for distal dendritic inputs. The higher integration time window for distal inputs was also reflected as high-amplitude wavelet coefficients in the spectrotemporal representation of the STA (Fig. $3 F$ ). Finally, increase in either the somatic $\bar{g}_{N a}$ (Fig. $3 H$ ) or $\bar{g}_{K D R}$ (Fig. 3I) increased $f_{\text {STA }}$ across somatodendritic locations (cf. Tables 2, 1).

Neuronal model with spiking dendrites exhibits weak spectral selectivity in spike initiation dynamics in the absence of $\mathrm{HCN}$ channels

Does the presence of spiking dendrites (Magee and Johnston, 1995; Gasparini et al., 2004; Gasparini and Magee, 2006; Losonczy and Magee, 2006; Bittner et al., 2012) alter spike initiation dynamics across somatodendritic locations? To answer this question, we introduced $\mathrm{Na}^{+}$and KDR channels with uniform density across the somatodendritic compartments of the balland-stick model and computed the STA measurements at various locations (Fig. 4). In striking contrast to our results with passive dendrites (Fig. $3 D-I$ ), here we found that $I_{S T A}^{P e a k}$ decreased and the spectrotemporal integration window of the STA was reduced for 
A

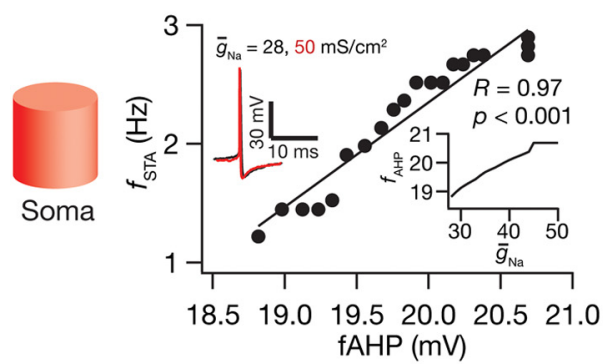

D

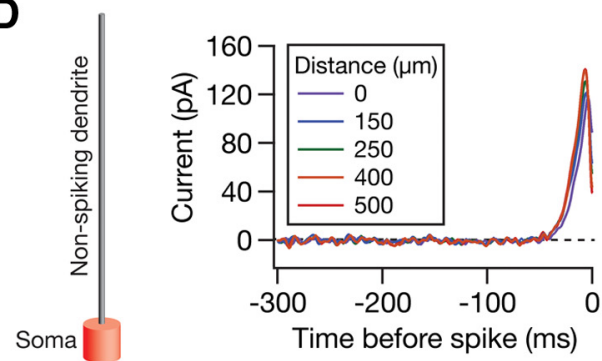

G

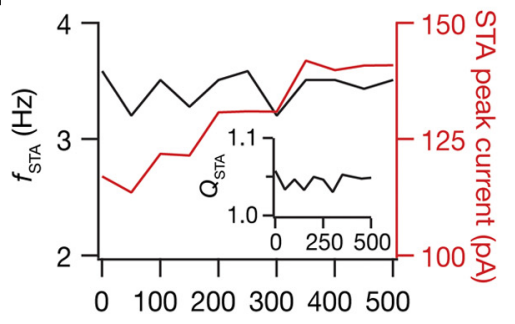

Distance from soma $(\mu \mathrm{m})$
B

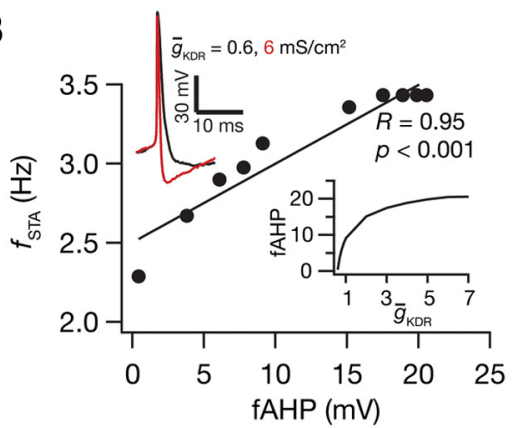

E

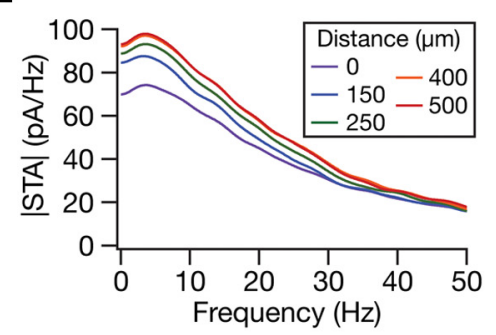

H

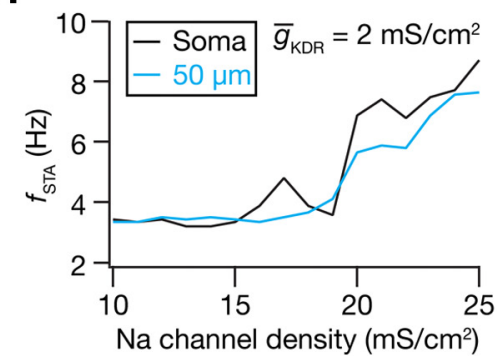

C

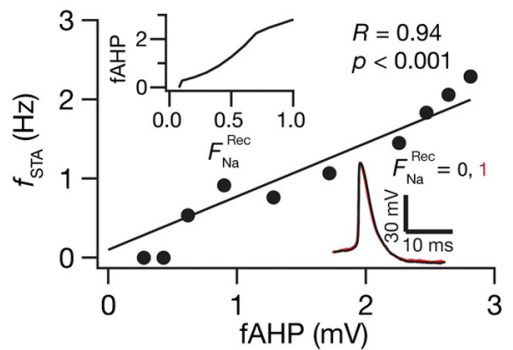

F

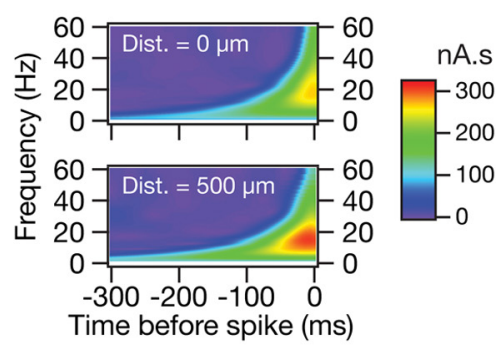

I

Figure 3. Spectral selectivity in spike initiation dynamics in the absence of HCN channels was in the delta frequency range. $\boldsymbol{A}$, Left, Depiction of single-compartmental model used for results presented in $\boldsymbol{A}-\boldsymbol{C}$. Right, $f_{\text {STA }}$ and fAHP, computed at different values of maximal Na channel density $\left(\bar{g}_{\mathrm{Na}}\right)$, exhibited a significantly strong correlation. Insets: Left, action potential traces obtained for two different values of $\bar{g}_{N a}$ showing a small variability in the fAHP across the tested range of $\bar{g}_{\mathrm{Na}}$. Right, fAHP as a function of $\bar{g}_{\mathrm{Na}}$, revealing this small variability. $\boldsymbol{B}, f_{\mathrm{STA}}$ and fAHP, computed at different values of maximal delayed rectifier $\mathrm{K}^{+}$channel density $\bar{g}_{K D R}$, exhibited a significantly strong correlation. Insets: Left, action potential traces obtained for two different values of $\bar{g}_{K D R}$ showing a large variability in the fAHP across the tested range of $\bar{g}_{K D R}$. Right, fAHP as a function of $\bar{g}_{K D R}$, revealing this large variability. $C$, $f_{\text {STA }}$ and fAHP, computed at different values of Na channel recovery factor $F_{r e c}^{N a}$, exhibited a significantly strong correlation. Insets: Left, fAHP as a function of $F_{r e c}^{N a}$ showing a small variability in fAHP across the entire range of $F_{r e c}^{N a}$. Right, Action potential traces obtained for two different values of $F_{r e c}^{N a}$ depicting this small variability. $\sigma_{\text {noise }}=0.6 \mathrm{nA}$ for $\boldsymbol{A}$ and $1.0 \mathrm{nA}$ for $\boldsymbol{B}, \boldsymbol{C}$. All correlation tests correspond to the Pearson's correlation test. $\boldsymbol{D}$, Left, Depiction of the ball-and-stick model consisting of a nonspiking dendrite used for results presented in $\boldsymbol{D}-\boldsymbol{F}$. $\boldsymbol{D}-\boldsymbol{F}$, Analyses of STA in passive dendrites. STA computed with the input GWN injected at different dendritic locations represented in the temporal $(\boldsymbol{D})$, spectral $(\boldsymbol{E})$, and the spectrotemporal $(\boldsymbol{F})$ domains. $\mathbf{G}, f_{\text {STA }}, Q_{\text {STA }}$ (inset) and $I_{S T A}^{P e a k}$ plotted as functions of the location of GWN injection (measured as the distance of the dendritic location from the soma). For $\boldsymbol{D}-\boldsymbol{G}$, values of $\bar{g}_{\mathrm{Na}}=12 \mathrm{mS} / \mathrm{cm}^{2}$ and $\bar{g}_{K D R}=2 \mathrm{mS} / \mathrm{cm}^{2}$. $\boldsymbol{H}, \boldsymbol{I}, f_{\text {STA }}$ plotted as functions of $\bar{g}_{N a}(\boldsymbol{H})$ and $\bar{g}_{K D R}(\boldsymbol{I}) ; \sigma_{\text {noise }}=1.2 \mathrm{nA}$. The plots are shown for STA computed when the input GWN was injected at the soma (black) and at a dendritic location $50 \mu \mathrm{m}$ away from the soma (cyan). Note that $f_{\mathrm{R}}$ was zero across all these simulations because $\bar{g}_{h}=0 \mathrm{~S} / \mathrm{cm}^{2}$.

distal dendritic inputs (Fig. 4A-D; cf. corresponding plots in Fig. $3)$. Analyses of somatic and dendritic traces with proximal versus distal inputs revealed the initiation and successful propagation of dendritic spikes (Gasparini et al., 2004; Gasparini and Magee, 2006) as the reason behind the reduction in $I_{S T A}^{P e a k}$ and in the integration time window. The non-monotonic nature of $I_{S T A}^{P e a k}$ (Fig. $4 D$ ) suggested a balance between the effects of passive filtering for inputs distal to the soma and the contribution of active conductances in initiating dendritic spikes at more distal locations where the excitability is higher. Despite these differences, the values of $f_{\text {STA }}$ across the somatodendritic locations (Fig. 4D) were similar to those in the passive case (Fig. $3 G$ ). It should be noted that the spatially confined bump (at the $400 \mu \mathrm{m}$ location) in $f_{\mathrm{STA}}$ in Figure $4 D$ was consequent to a weak maxima in an almost flat $|\operatorname{STA}(f)|$ (Fig. 4B). The flat nature of the Fourier spectra was also reflected as very low values of $Q_{\mathrm{STA}}$, implying weak spectral selectivity in spike initiation dynamics spanning all somatodendritic locations
(Fig. 4D). Finally, the dependence of $f_{\mathrm{STA}}$ on $\bar{g}_{\mathrm{Na}}$ (Fig. $4 E$ ) and $\bar{g}_{K D R}$ (Fig. $4 F$ ) were qualitatively similar to our results with a passive dendrite (Fig. $3 H, I$ ).

HCN channels normalize the STA characteristic frequency to the theta-frequency range across the dendritic arbor

How does frequency selectivity in spike initiation dynamics vary with the insertion of HCN channels into the somatodendritic compartments? To answer this question, we inserted HCN channels with an experimentally constrained (Magee, 1998; Lörincz et al., 2002) somatodendritic gradient in $\bar{g}_{h}$ (Equation 7; Fig. $5 A$ ) into the previous model where $\mathrm{Na}^{+}$and KDR channels were present only in the soma (Fig. 3D-I). The gradient was set such that local $\left(f_{\mathrm{R}}\right.$; Fig. $\left.5 B, D\right)$ and transfer $\left(f_{\mathrm{TR}}\right.$; Fig. $\left.5 C, D\right)$ resonance frequencies, as functions of distance from the soma, matched with corresponding experimental values (Narayanan and Johnston, 2007; Hu et al., 2009; Vaidya and Johnston, 2013). We set 
Table 2. Sensitivity analysis for STA measurements corresponding to various passive and active parameters in the ball-and-stick model without HCN channels

\begin{tabular}{|c|c|c|c|c|c|}
\hline & Parameters & Tested range & $I_{S T A}^{P e a k}$ & $f_{\text {STA }}$ & $Q_{\text {STA }}$ \\
\hline \multirow[t]{7}{*}{ Nonspiking dendrite } & Baseline & $0-500$ & Increases & Decreases & Decreases \\
\hline & $\bar{g}_{\mathrm{Na}}$ & $10-24$ & Decreases & Increases & Increases \\
\hline & $\bar{g}_{K D R}$ & $1-5$ & Increases & Increases & Decreases \\
\hline & $R_{\mathrm{m}}$ & $8-16$ & Decreases & No change & No change \\
\hline & $R_{\mathrm{a}}$ & $90-140$ & Decreases & No change & No change \\
\hline & $C_{m}^{d}$ & $0.8-1.2$ & Decreases & Decreases & Decreases \\
\hline & $I_{S T A}^{P}$ Pak & $0-1$ & Decreases & Increases & Increases \\
\hline \multirow[t]{7}{*}{ Spiking dendrite } & Baseline & $0-500$ & Bell-shaped & Increases & Increases \\
\hline & $\bar{g}_{\mathrm{Na}}$ & $10-24$ & Decreases & Increases & Increases \\
\hline & $\bar{g}_{K D R}$ & $1-5$ & Increases & Increases & Decreases \\
\hline & $R_{\mathrm{m}}$ & $8-16$ & Decreases & No change & No change \\
\hline & $R_{a}$ & $90-140$ & Non-monotonic ${ }^{a}$ & Increases (>200 $\mu \mathrm{m})$ & Increases (>200 $\mu \mathrm{m})$ \\
\hline & $C_{m}^{d}$ & $0.8-1.2$ & Increases & Decreases & Decreases \\
\hline & $I_{S T A}^{P e a k}$ & $0-1$ & Decreases & Increases & Decreases \\
\hline
\end{tabular}

Shown are changes in the STA peak, STA characteristic frequency, and STA selectivity strength obtained from simulations in which each of the multicompartmental parametric values were increased. Rows against "Baseline" parametric values refer to the dependence of the respective STA measurements as a function of the location of GWN injection. The other rows represent changes in STA measurements with reference to the "Baseline" values.

${ }^{a}$ Increases perisomatically and decreases distally $(>100 \mu \mathrm{m})$ with increase in $R_{\mathrm{a}}(\Omega \cdot \mathrm{cm})$.

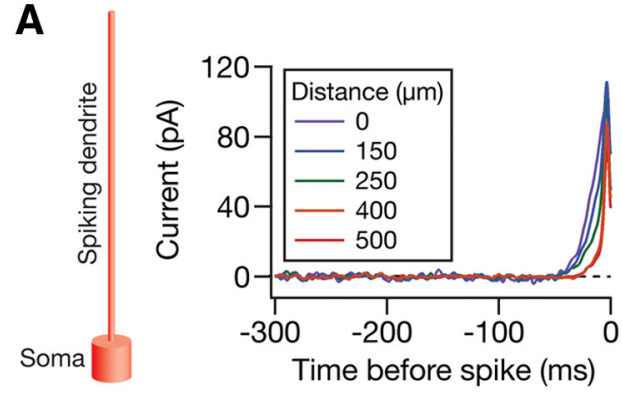

D

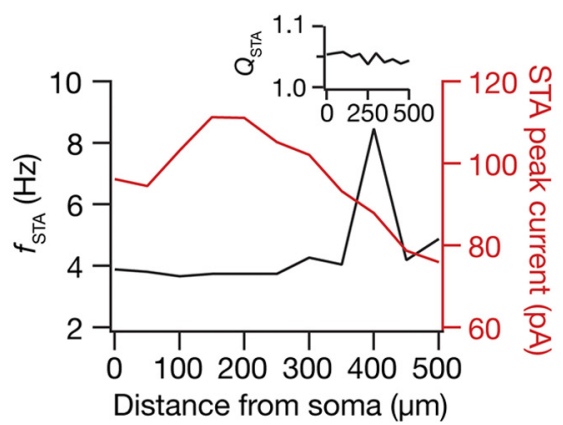

B

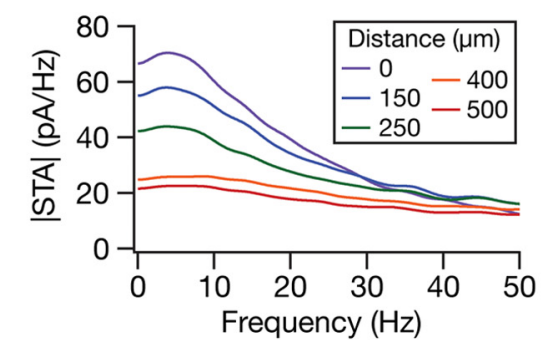

E

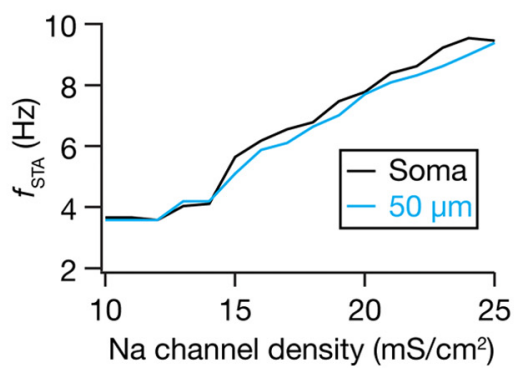

C

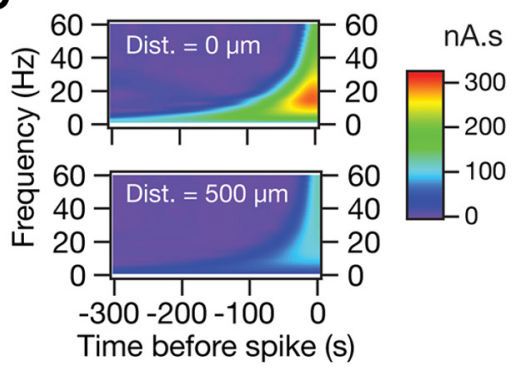

$\mathbf{F}$

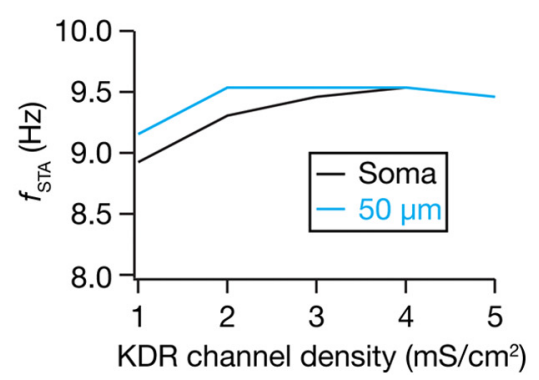

Figure 4. Weak spectral selectivity in spike initiation dynamics of a neuronal model with a spiking dendrite. $\boldsymbol{A}$, Left, Depiction of the ball-and-stick model consisting of a spiking dendrite used for all results presented in this figure. $\boldsymbol{A}, \boldsymbol{B}$, Analyses of STA in spiking dendrites, with $\mathrm{Na}^{+}$and KDR channels uniformly distributed across the somatodendritic axis. STA computed with the input GWN injected at different dendritic locations represented in the temporal $(\boldsymbol{A})$, the spectral $(\boldsymbol{B})$, and the spectrotemporal ( $\boldsymbol{C}$ ) domains. $\boldsymbol{D}, f_{\mathrm{STA}}, \mathrm{Q}_{\mathrm{STA}}$ (inset), and $I_{S T A}^{P e a k}$ plotted as functions of the location of GWN injection (measured as the distance of the dendritic location from the soma). For $\boldsymbol{A}-\boldsymbol{D}$, values of $\bar{g}_{\mathrm{Na}}=12 \mathrm{mS} / \mathrm{cm}^{2}$ and $\bar{g}_{K D R}=2 \mathrm{mS} / \mathrm{cm}^{2} . \boldsymbol{E}, \boldsymbol{F}, f_{\text {STA }}$ plotted as functions of $\bar{g}_{N a}(\boldsymbol{E})$ and $\bar{g}_{K D R}(\boldsymbol{F}) ; \sigma_{\text {noise }}=1.2 \mathrm{nA}$. The plots are shown for STA computed when the input GWN was injected at the soma (black) and at a dendritic location $50 \mu \mathrm{m}$ away from the soma (cyan). $\bar{g}_{h}=0 \mathrm{~S} / \mathrm{cm}^{2}$ for all simulations.

$\sigma_{\text {noise }}$ for each somatodendritic location such that somatic response (firing frequency) to GWN injection was sparse and location independent (Magee and Cook, 2000; Andrasfalvy and Magee, 2001). As expected from previous results (Figs. 1, 2), with the introduction of HCN channels, the STA switched to prominently displaying characteristics of a coincidence detector with strong theta selectivity regardless of the location of GWN injection (Fig. 5E). Furthermore, the integration window of the STA increased with increase in distance from the soma and was accompanied by a distance-dependent increase in $I_{S T A}^{P e a k}$ and the wavelet coefficient amplitudes (cf. Figs. 5E, F, 3D, F).

Surprisingly, we found $f_{\mathrm{STA}}$ to be normalized across the somatodendritic span (Fig. 5G), suggesting that the spike initiation dynamics were optimally responding to one specific frequency across the entire neuronal structure regardless of input location.
Further, upon increasing $g_{h}^{\text {Base }}$ (Equation 7), and thereby increasing $\bar{g}_{h}$ throughout the somatodendritic span, we found that $f_{\text {STA }}$ increased to higher values (Fig. $1 D$ ), with location-invariance still intact. Interestingly, whereas STA selectivity in the absence of $\mathrm{HCN}$ channels was weak (Fig. $3 G$ ), the insertion of $\mathrm{HCN}$ channels increased $Q_{\text {STA }}$ (Fig. 5H). Finally, introduction of constant densities of HCN channel at the soma or along the entire somatodendritic span also normalized the $f_{\mathrm{STA}}$, although requiring higher $\bar{g}_{h}$ (than the $g_{h}^{\text {Base }}$ in the sigmoidal gradient) or a linear gradient (Equation 8) resulted in $f_{\mathrm{STA}}$ and $Q_{\mathrm{STA}}$ values that were not significantly different from those achieved by the sigmoidal gradient. In summary, when measured with location-dependent $\sigma_{\text {noise }}$ to achieve normalized firing frequency, we found that $f_{\text {STA }}$ and $Q_{\mathrm{STA}}$ were normalized across the dendritic arbor in the presence of HCN channels regardless of their exact somatodendritic 
A

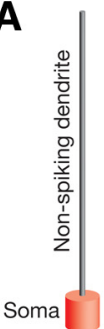

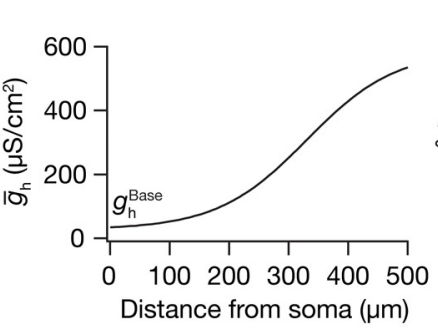

D

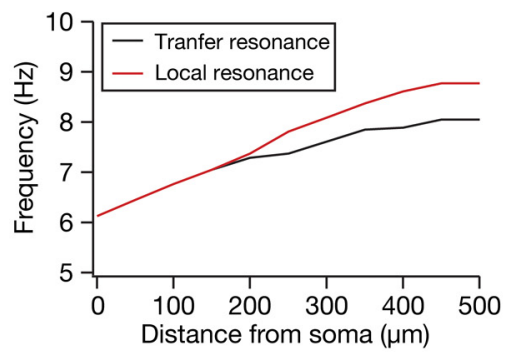

G

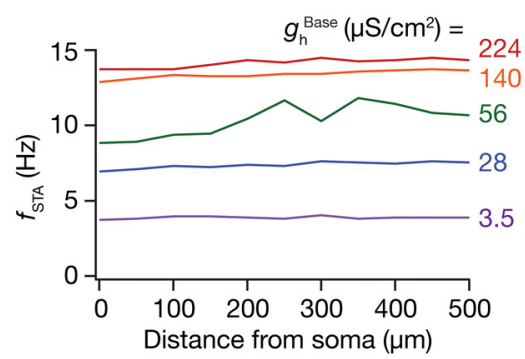

B

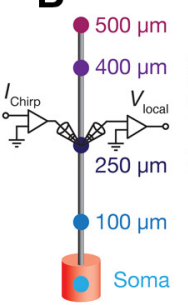

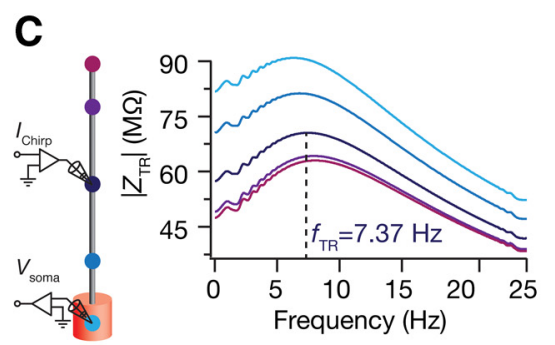

E

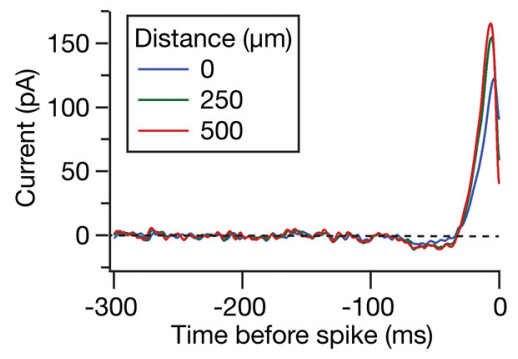

H

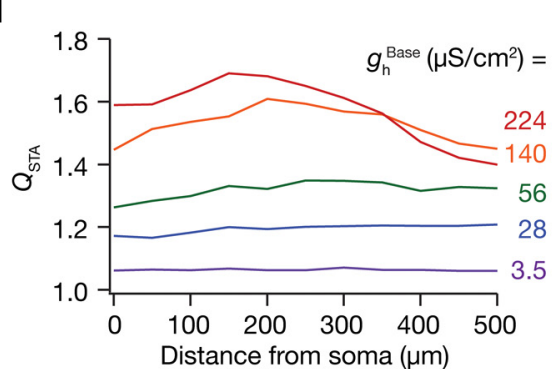

F

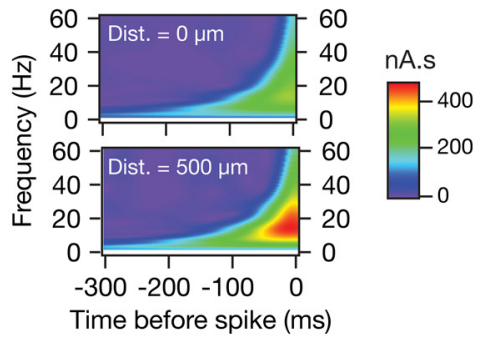

I

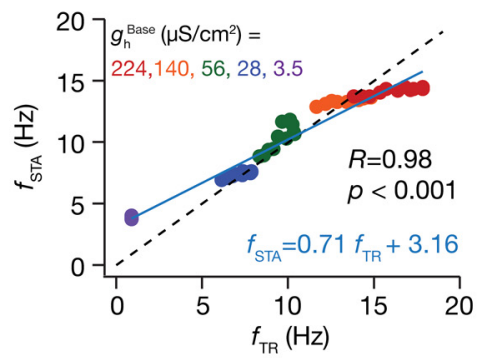

Figure 5. Spatial gradient in HCN channel density rendered the STA characteristic frequency location-invariant in neurons with nonspiking dendrites. $A$, Left, Depiction of the ball-and-stick model consisting of a nonspiking dendrite used for all results presented in this figure. Right, Spatial gradient in HCN channel density (Equation 7). B, Left, Illustration of measurement of local resonance frequency by applying a chirp current $\left(I_{\text {chirp }}\right)$ at a somatodendritic location and measuring the voltage response at the same location $\left(V_{\text {local }}\right)$. Right, Local impedance amplitude $\left(\left|Z_{\text {local }}\right|\right)$ profiles plotted for different locations (color coded by dots on the left). Depicted local resonance frequency $\left(f_{\mathrm{R}}\right)$ corresponds to the $250 \mu \mathrm{m} \mathrm{location}$. C, Left, Illustration of measurement of transfer resonance frequency by applying a chirp current $\left(I_{\text {Chirp }}\right)$ at a somatodendritic location and measuring the voltage response at the somatic location $\left(V_{\text {soma }}\right)$. Right, Transfer impedance amplitude $\left(\left|Z_{T R}\right|\right)$ profiles plotted for different locations (color coded by dots on the left). Depicted transfer resonance frequency $\left(f_{\mathrm{TR}}\right)$ corresponds to the $250 \mu \mathrm{m}$ location. $\boldsymbol{D}$, Local and transfer resonance frequencies plotted as functions of distance from the soma. $\boldsymbol{E}, \boldsymbol{F}$, Analyses of STA in nonspiking dendrites. STA computed with the input GWN injected at different dendritic locations represented in the temporal $(\boldsymbol{E})$ and the spectrotemporal $(\boldsymbol{F})$ domains. $\boldsymbol{G}, \boldsymbol{H}, f_{\text {STA }}(\boldsymbol{G})$ and $Q_{\text {STA }}(\boldsymbol{H})$ plotted as functions of the location of $G W N$ injection (measured as the distance of the dendritic location from the soma) illustrate its location-invariance across different values of $g_{h}^{\text {Base }}$ (Equation 7$) . I, f_{\text {STA }}$ and $f_{\text {TR, }}$ computed for different base values of HCN channel density at each dendritic location of GWN injection, exhibited a significantly strong correlation (Pearson's correlation test) for model neurons with nonspiking dendrites. The dashed line corresponds to the $45^{\circ}$ line and the solid line represents a linear fit.

distribution. The exact values of these two measures, however, seemed to be determined by the overall numbers of HCN channels rather than by their exact distribution (Angelo et al., 2007).

\section{STA characteristic frequency is strongly related to frequency selectivity in subthreshold transfer impedance measured across neuronal compartments}

We had noted that STA measurements and their sensitivities to parametric variations were linked to specific measurements related to subthreshold frequency selectivity in the singlecompartmental model (Table 1; Narayanan and Johnston, 2008). In the ball-and-stick model, we were injecting the GWN current at a dendritic or a somatic location, with voltage recordings always fixed at the soma. Given this configuration, we reasoned that subthreshold transfer impedance properties (Ulrich, 2002; Cook et al., 2007; Hu et al., 2009; Vaidya and Johnston, 2013) were putative subthreshold correlates for spectral selectivity in the spike initiation dynamics measured across the somatodendritic compartments. We quantified several transfer-resonancerelated measurements (see Materials and Methods) and assessed their relationships to STA measurements. We found that $f_{\mathrm{TR}}$ and $f_{\text {STA }}$, computed at different locations and across different HCN channel densities, were significantly and strongly correlated with each other (Fig. 5I). Furthermore, when we compared the sensitivities of $f_{\mathrm{TR}}$ and $f_{\mathrm{STA}}$ across several parameters, we found a strong relationship in their dependencies on these parameters (Tables 3, 4). We also assessed $I_{S T A}^{P e a k}$ and $Q_{S T A}$ and found them to be strongly related to $\left|Z_{\mathrm{TR}}\right|_{\max }$ (inverse) and $Q_{\mathrm{TR}}$, respectively, including dependencies on underlying active and passive parameters (Tables 3, 4).

Neuronal model with spiking dendrites reduces the spike integration window and increases the STA characteristic frequency in the presence of $\mathrm{HCN}$ channels

How do spiking dendrites alter theta-frequency selectivity in the location-dependent spike initiation dynamics in the presence of somatodendritic HCN channels? To answer this question, we inserted $\mathrm{Na}^{+}$and KDR channels uniformly across the somatodendritic compartments on top of the HCN channel gradient. The HCN channel density (Fig. $5 A$ ), the subthreshold $f_{\mathrm{R}}$ (Fig. $5 B, D$ ), and $f_{\mathrm{TR}}$ (Fig. $5 C, D$ ) remained the same as our simulations with the nonspiking dendrite. In contrast to the case with non- 
Table 3. Sensitivity analysis for STA measurements corresponding to various passive and active parameters in the ball-and-stick model in the presence of a sigmoidal gradient in HCN channels

\begin{tabular}{|c|c|c|c|c|c|}
\hline & Parameters & Tested range & $I_{S T A}^{P e a k}$ & $f_{\text {STA }}$ & $Q_{\text {STA }}$ \\
\hline \multirow[t]{10}{*}{ Nonspiking dendrite } & Baseline & $0-500$ & Increases & Normalized & Normalized \\
\hline & $\bar{g}_{h}$ & $3.5-224$ & Increases & Increases & Increases \\
\hline & $\bar{g}_{N a}$ & $12-24$ & Decreases & Increases & Increases \\
\hline & $\bar{g}_{K D R}$ & $1-8$ & Increases & Increases & Decreases \\
\hline & $R_{\mathrm{m}}$ & $8-16$ & Decreases & Increases & Increases \\
\hline & $R_{\mathrm{a}}$ & $80-120$ & No change & Decreases & Decreases \\
\hline & $C_{m}$ & $0.6-1.2$ & Decreases & Decreases & Decreases \\
\hline & $V_{1 / 2}(\mathrm{HCN})$ & -100 to -40 & Increases & Bell-shaped & Bell-shaped \\
\hline & $\tau_{\mathrm{HCN}}$ & $3-66$ & Decreases & Decreases after a threshold & Asymmetric bell-shaped \\
\hline & $I_{S T A}^{P e a k}$ & $0-1$ & Decreases & Increases & Increases \\
\hline \multirow[t]{10}{*}{ Spiking dendrite } & Baseline & $0-500$ & Bell-shaped & Normalized & Normalized \\
\hline & $\bar{g}_{h}$ & $3.5-224$ & Increases & Increases & Increases \\
\hline & $\bar{g}_{N a}$ & $12-21$ & Decreases & Increases & Increases \\
\hline & $\bar{g}_{K D R}$ & $1-5$ & Increases & Increases & Decreases \\
\hline & $R_{\mathrm{m}}$ & $8-16$ & Decreases & Decreases & Increases \\
\hline & $R_{\mathrm{a}}$ & $80-120$ & Non-monotonic ${ }^{a}$ & Non-monotonic ${ }^{a}$ & Non-monotonic ${ }^{a, b}$ \\
\hline & & $0.6-1.4$ & Increases & Decreases & Decreases \\
\hline & $V_{1 / 2}(\mathrm{HCN})$ & -100 to -40 & Increases & Bell-shaped & Bell-shaped \\
\hline & $\tau_{\mathrm{HCN}}$ & $3-66$ & Decreases & Decreases after threshold & Asymmetric bell-shaped \\
\hline & $I_{S T A}^{P e a k}$ & $0-1$ & Decreases & Increases & Increases \\
\hline
\end{tabular}

Shown are changes in the STA peak current, STA characteristic frequency, and STA selectivity strength obtained from simulations in which each of the multicompartmental parametric values were increased. Rows against "Baseline" parametric values refer to the dependence of the respective STA measurements as a function of the location of GWN injection. The other rows represent changes in STA measurements with reference to the "Baseline" values.

ancreases perisomatically and decreases distally $(>100 \mu \mathrm{m})$ with increase in $R_{\mathrm{a}}$.

${ }^{b}$ Small changes.

Table 4. Sensitivity analyses of transfer resonance related measurements for inputs at a dendritic trunk location $250 \mu \mathrm{m}$ away from the soma

\begin{tabular}{|c|c|c|c|c|}
\hline \multirow[b]{2}{*}{ Increase in } & \multirow[b]{2}{*}{ Tested range } & \multicolumn{3}{|l|}{ Effects on } \\
\hline & & $f_{\mathrm{TR}}$ & $Q_{T R}$ & $\left|Z_{\mathrm{TR}}\right|_{\max }$ \\
\hline$V_{\mathrm{m}}$ & -120 to -50 & Bell-shaped & Bell-shaped & Decreases \\
\hline$R_{\mathrm{m}}$ & $8-17$ & Decreases & Increases $^{b}$ & Increases \\
\hline$C_{m}$ & $0.5-1.5$ & Decreases & Decreases & Decreases \\
\hline$R_{\mathrm{a}}$ & $50-140$ & Non-monotic ${ }^{a}$ & Non-monotic ${ }^{a}$ & Non-monotic \\
\hline $\bar{g}_{h}$ & $3.5-224$ & Increases & Increases & Decreases \\
\hline$V_{1 / 2}$ & -120 to 0 & Bell-shaped & Bell-shaped & Decreases sigmoidally \\
\hline$\tau_{\mathrm{HCN}}$ & $3-66$ & Asymmetric bell-shaped & Asymmetric bell-shaped & Asymmetric bell-shaped \\
\hline
\end{tabular}

${ }^{a}$ Decreases perisomatically and increases distally $(>200 \mu \mathrm{m})$ with increase in $R_{\mathrm{a}}$.

${ }^{b}$ Small changes.

Increases perisomatically and decreases distally $(>100 \mu \mathrm{m})$ with increase in $R_{\mathrm{a}}$.

spiking dendrites (Fig. 5), both $I_{S T A}^{P e a k}$ and the spike integration window reduced with increase in distance of the GWN injection (Fig. 6A, B), consequent to the initiation and successful propagation of dendritic spikes. However, scaling the $\bar{g}_{h}$ gradient in the spiking dendrite still resulted in scaling $f_{\mathrm{STA}}$ and $Q_{\mathrm{STA}}$ without perturbing the distance-invariant normalization (Fig. $6 C, D$ ). Importantly, the actual values of $f_{\mathrm{STA}}$ and $Q_{\mathrm{STA}}$ were higher in the model with a spiking dendrite (cf. Figs. $5 G, H, 6 C, D$ ), suggesting stronger frequency selectivity in the STA at frequencies higher than those with nonspiking dendrite. Further, we found a significant and strong correlation between $f_{\mathrm{STA}}$ and $f_{\mathrm{TR}}$ (Fig. $6 E$ ), although the deviation from the equivalence line $\left(45^{\circ}\right)$ was higher in the spiking dendrite than in the nonspiking dendrite (cf. Figs. $5 I, 6 E)$. This deviation was consequent to the dependencies of $f_{\mathrm{STA}}\left(\right.$ and not $f_{\mathrm{TR}}$ ) on signal variance and on spiking-related conductances (Table 3), which were similar to those in the singlecompartmental model (Fig. 2, Tables 1,2). Finally, the fAHP of the model with a spiking dendrite was significantly higher $(\sim 8-9$ $\mathrm{mV}$ at the soma) than the fAHP of a nonspiking dendrite $(\sim 1-2$ $\mathrm{mV}$ at the soma), thus confirming the correlation between fAHP and $f_{\mathrm{STA}}$ in dendritic models as well (Fig. 3).
Morphologically realistic model expressing HCN channels exhibits location-dependent spike initiation dynamics with theta-frequency selectivity

Thus far, we had arrived at specific roles for channel kinetics and voltage dependencies (Figs. 1, 2, 3) and the role of dendrites and their ion channels (Figs. 3, 4, 5, 6) in defining and altering spike initiation dynamics. How do these findings translate in a conductance-based, morphologically precise neuronal model with experimentally constrained channel properties and physiological measurements? To answer this question, we built a morphologically precise model with experimentally constrained gradients of active and passive properties, which set the values $R_{\text {in }}, f_{\mathrm{R}}$, and $f_{\mathrm{TR}}$ along the somatoapical trunk (Fig. 7A-C) to physiologically comparable values. As $f_{\mathrm{STA}}$ and somatic firing frequency increased with $\sigma_{\text {noise }}$ regardless of location of GWN injection, we varied $\sigma_{\text {noise }}$ across locations such that the somatic firing rate was sparse and normalized for inputs at any location. We computed STA across the somatoapical trunk of the model by injecting GWN current at several somato-apical trunk locations and measuring somatic spike timings. Strikingly, the spike integration time window reduced with an increase in the location of 
GWN current injection (Fig. 7D; note the distinct change in shape of the STA for dendritic input locations $>\sim 250 \mu \mathrm{m}$ from the soma), reflecting also in the spectrotemporal spread of the STA (Fig. $7 E$ ). In contrast to our results with a spiking dendrite in a ball-and-stick model (Fig. 6), $I_{S T A}^{\text {Peak }}$ increased as the GWN current was injected at more distal locations (Fig. $7 F$ ). This was a direct reflection of the reduced distal excitability consequent to an experimentally matched decrease in $R_{\text {in }}$ along the somatoapical axis (Narayanan and Johnston, 2007) of the morphologically realistic model (Fig. 7C). Conversely, in Figure 6, the model involved uniform passive properties, thereby reducing $I_{S T A}^{\text {Peak }}$ at distal locations because of increased distal excitability (the larger somatic surface area and the axonal load [Debanne et al., 2011] reduces somatic excitability). Despite this, and consequent to the presence of dendritic $\mathrm{Na}^{+}$and $\mathrm{KDR}$ channels, dendritic spikes were elicited for distal current injections (beyond $\sim 250$ $\mu \mathrm{m})$, leading to a reduction in the spectrotemporal spike integration window (Fig. $7 D, E)$. Further, $f_{\mathrm{STA}}$ and $f_{\mathrm{TR}}$ were in the theta-frequency range (Fig. $7 F$ ), with a significant and strong correlation between the two measurements (Fig. 7G) across the somato-apical trunk.

To further confirm that the location dependence of the STA was concurrent with a physiologically relevant response of the neuron, noisy suprathreshold sinusoidal inputs of various frequencies were injected at different locations along the dendrite and the suprathreshold resonance frequency $\left(f_{\mathrm{SR}}\right)$ was quantified. $f_{\mathrm{SR}}$ was dependent on the location of input current injection, but increased with increase in sinusoidal amplitude regardless of the location (Fig. $7 \mathrm{H}, \mathrm{I}$ ), mirroring the location dependence of $f_{\text {STA }}$ and its relationship with input strength. Finally, when sinusoid amplitudes at different dendritic locations were adjusted so as to obtain normalized peak firing frequency at the soma (Fig. 7J; to match with the normalization of $\sigma_{\text {noise }}$ in computing $f_{\text {STA }}$ in Fig. $7 F$ ), the $f_{\text {SR }}$ also became distance invariant and was comparable to the normalized $f_{\mathrm{STA}}$ and the $f_{\mathrm{TR}}$ (Fig. $7 \mathrm{~K}$ ). In summary, in a neuron with dendrites, subthreshold transfer impedance properties had a significant influence on the strong theta-frequency spectral selectivity in the location-dependent STA, which also manifested as a frequency preference for firing response of the neuron. Importantly, location invariance of $f_{\mathrm{STA}}$ could be achieved in spiking or nonspiking dendrites through an "active" normalization process (Magee and Cook, 2000; Andrasfalvy and Magee, 2001) that is mediated by tight interactions among various active and passive mechanisms (Table 1, 2, 3, 4).

Dynamically reconfigurable multi-STA model can account for the location dependence of spike initiation dynamics in neurons with plastic active dendrites

The STA is generally thought of as a linear representation of the neuronal input-output relationship, within a linear-nonlinear

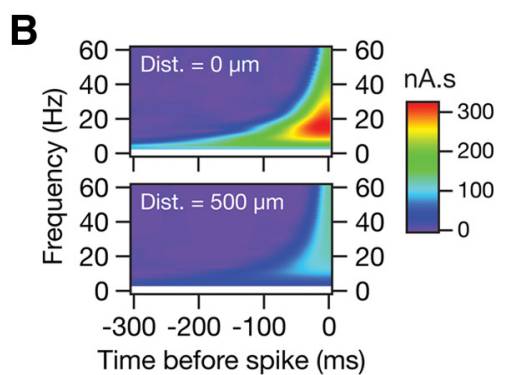

D
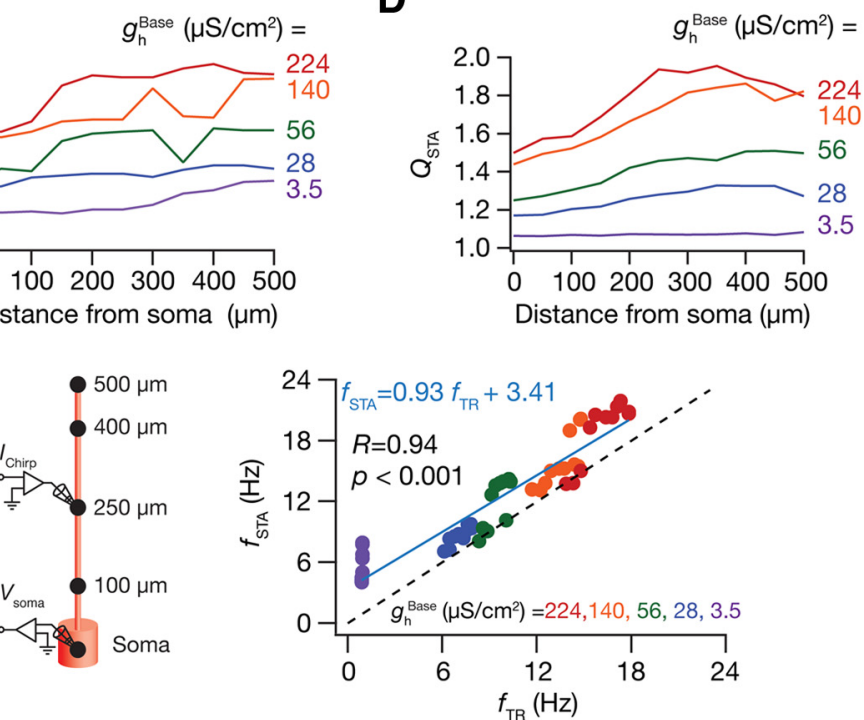

Figure 6. Spatial gradient in HCN channel density rendered the STA characteristic frequency location invariant in neurons with spiking dendrites. The spatial gradient in HCN channel density and the corresponding subthreshold $f_{\mathrm{R}}$ and $f_{\mathrm{TR}}$ were the same as in Figure $5 A-D$. A 列 (D) pponctions of the location of GWN injection (measured as the distance of the dendritic location from the soma) illustrate its cation invariance across different values of $g_{h}^{\text {Base }}$. $\boldsymbol{E}, f_{\mathrm{STA}}$ and $f_{\mathrm{TR}^{\prime}}$ computed for different base values of HCN channel density at each dendritic location of GWN injection exhibited a significantly strong correlation (Pearson's correlation test) for model neurons with spiking dendrites. The dashed line corresponds to the $45^{\circ}$ line and the solid line represents a linear fit.

(LN) framework. Under this framework, an implicit assumption is that a neuron is characterized by a single input-output relationship that embodies the features to which the neuron responds (Hong et al., 2007; Prescott et al., 2008a; Hong et al., 2012; Ratté et al., 2013). Our results delineating the location dependence of spike initiation dynamics in neurons with dendrites demonstrate that, in a multi-input-single-output (MISO) system like that of a single neuron, the proposition that a single linear kernel defines the entire neuron is clearly inadequate. This inadequacy is further exacerbated by our findings that the STA is heavily dependent on the active and passive properties of the dendrites, apart from being modulated by location-dependent passive filtering. Combined with the experimentally well established forms of plasticity in various dendritic ion channels and their properties, it is clear that the neuronal input-output properties are far more complex and dynamic than what could be captured by a single linear kernel. So, how do we characterize the input-output relationships of a neuron with complex morphology quantitatively? How do we account for the presence of various kernels defining a single neuron, which are dynamically dependent on the location of signal origin, the local state of the neuronal compartment, the input statistics, and the various active and passive properties? 
A

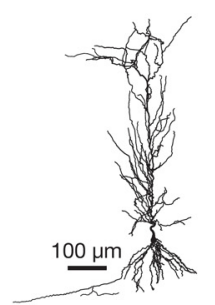

B

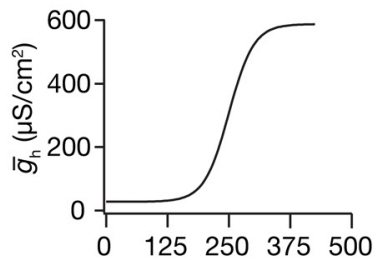

Radial distance from soma $(\mu \mathrm{m})$
C

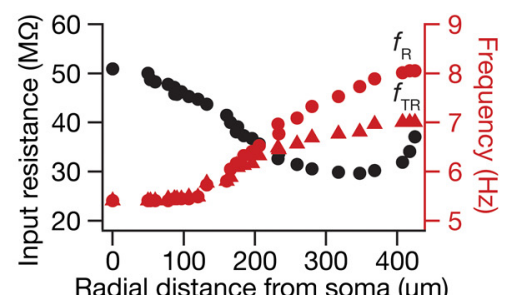

F

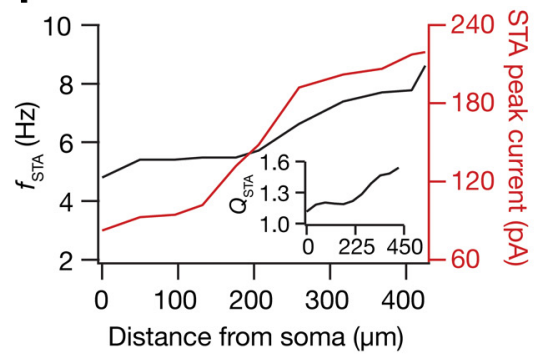

J

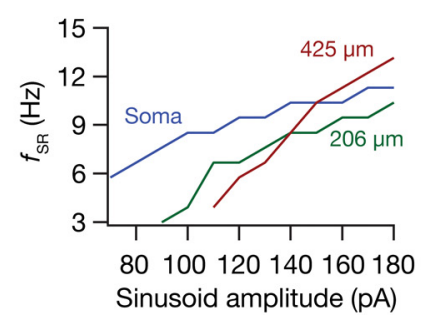

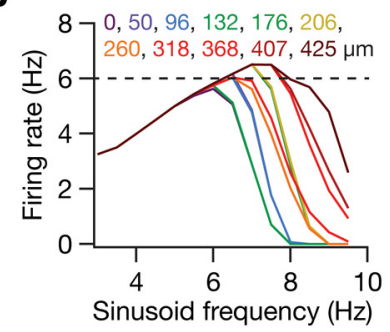

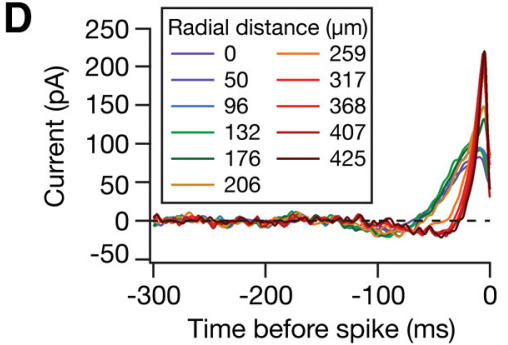

G

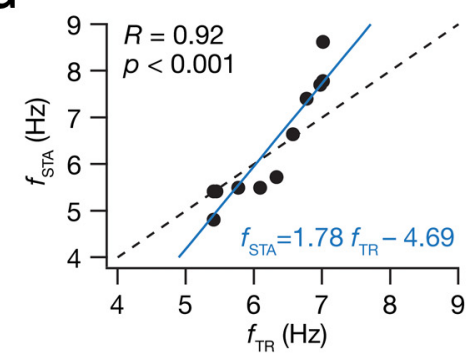

K

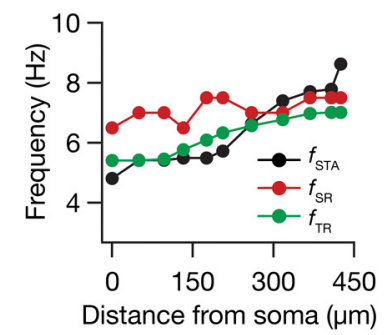

Figure 7. Experimentally constrained gradient in HCN channel density results in spatially widespread theta-frequency selectivity in the spike initiation dynamics. $\boldsymbol{A}$ - $\boldsymbol{C}$, Morphologically realistic model used for all results presented in this figure $(\boldsymbol{A})$ with a sigmoidal gradient in $\mathrm{HCN}$ channel density $(\boldsymbol{B})$ matched input resistance $\left(R_{\text {in }}\right)$, local $\left(f_{R}\right)$, and transfer $\left(f_{\mathrm{TR}}\right)$ resonance frequencies $(\boldsymbol{C})$ from experimental observations. $\boldsymbol{D}, \boldsymbol{E}$, STA computed with the input $\mathrm{GWN}$ injected at different dendritic locations represented in the temporal $(\boldsymbol{D})$ and the spectrotemporal domains $(\boldsymbol{E}) . \boldsymbol{F}, f_{\mathrm{STA}}, \boldsymbol{Q}_{\mathrm{STA}}($ inset), and $I_{S T A}^{P e a k}$ plotted as functions of the location of GWN injection (measured as the distance of the dendritic location on the trunk from the soma). $G, f_{S_{T A}}$ and $f_{\text {TR' }}$, computed at different locations of GWN injection, exhibited a significantly strong correlation (Pearson's correlation test). $\boldsymbol{H}$, Neuronal firing rate plotted as a function of the frequency of sinusoidal current injected at the soma. The dependence of the neuronal firing rate on the sinusoidal amplitude is also shown. $I$, Suprathreshold resonance frequency $\left(f_{S R}\right)$ plotted as a function of amplitude shown for three different locations of sinusoidal current injection. The values for the somatic locations were derived from plots shown in $\boldsymbol{H} . \boldsymbol{J}, \boldsymbol{K}$, Normalization of somatic peak firing frequency across dendritic locations of input-current injection $(J)$ resulted in distance-dependent normalization of the $f_{S R}$ consistent with the normalization of subthreshold transfer resonance frequency $\left(f_{\text {TR }}\right)$ and the STA characteristic frequency $\left(f_{\text {STA }}\right)(K)$. The values for $f_{\text {TR }}$ are from $\boldsymbol{C}$ and for $f_{\text {STA }}$ are from $\boldsymbol{F}$.

In answering these questions, we noted that the computation of spike initiation dynamics with reference to any location required, by definition, that the spike be elicited by inputs only to that location. This implied that the location-dependent STA in a pyramidal neuron defines spike initiation dynamics in response to multiple synchronous inputs converging onto that dendritic location. A large body of experimental evidence points to the existence of and the necessity for such synchronous inputs converging onto a dendritic branch (Gasparini et al., 2004; Gasparini and Magee, 2006; Buzsáki, 2010; Magee, 2011; Lisman and Jensen, 2013). Against this background, the location-dependent STA forms an important tool for the analyses of neuronal inputoutput characteristics when synchronous inputs converge onto dendritic locations.

Whereas this analysis would fit into a scenario where neuronal spiking is governed by synchronous inputs arriving at one single location (coincident-detector mode), how do we reconcile the role of location-dependent STA under a scenario in which inputs from multiple locations integrate together (integrator mode) to elicit a somatic spike? By definition, the STA computed at a given location serves as a linear kernel characterizing spike initiation dynamics for that location. Accounting for the STA being location dependent, we present a scenario in which the dendritic arbor could be discretized into several dendritic segments such that the STA remains largely invariant across locations within each segment, but varies across segments. Now, the synapses that converge on to each of these segments could be clustered such that signal through these synapses collectively pass through a linear filter that is defined by the STA for that specific dendritic segment. Finally, the output of linear filters associated with all of the dendritic segments could be combined (linearly or nonlinearly) and presented to an axosomatic nonlinearity, which would generate the spike (Fig. $8 A$ ). Note that this model is a straightforward MISO extension to the standard model built of a linear kernel followed by a decision nonlinearity (Rieke et al., 1999; Dayan and Abbott, 2005). The advantage that this extension presents is that it accounts for the input dependence of the STA in the MISO system, and each of these STAs could be independently and dynamically reconfigured based on the state of the neuron, the passive and active properties associated with the individual segments, and input statistics.

How do we quantify the STA of individual segments with the constraint that the characterization should be capable of spanning the range of all the STA measurements, including spike integration window, observed across our sensitivity analyses? We arrived at a mathematical expression for the STA of a neuron and 
argue that this quantitative characterization can be used as an abstraction of spectral selectivity in the spike initiation dynamics. Specifically, we present the cosine-modulated exponential (CosExp) function (Equation 14) as an abstraction for the STA of the individual dendritic segments, the parameters of which can cover the variability that we observed in our simulations spanning different models (Figs. 1, 2, 3, 4, 5, 6, 7) as follows:

$$
\begin{array}{r}
\operatorname{Cos} \operatorname{Exp}(t)=B_{f i t}+A_{f i t} \cos \left(2 \pi f_{f i t} t\right. \\
\left.+\phi_{f i t}\right) \exp \left(-\frac{\mathrm{t}}{\tau_{\text {fit }}}\right)
\end{array}
$$

To test the efficacy of this function in defining STA of varied shapes, we used different densities of HCN channels and fit the corresponding STA traces with this CosExp (Fig. $8 A, B$ ). We chose $\bar{g}_{h}$ because the STAs associated with changes in $\bar{g}_{h}$ spanned a wide range (Figs. 1, 2), including the switch from an integrator-type STA to a coincidence detector-type STA. We found the CosExp function to be versatile in accounting for the variabilities in the STA and found that each of the fit parameters of the CosExp function translated to a physiologically relevant STA metric. Quantitatively, $f_{\text {fit }}$ (Fig. $8 D$ ) was similar to $f_{\text {STA }}$ (Fig. $1 D), \tau_{\text {fit }}$ defined the spike integration time window (Fig. $8 E$ ), and $A_{\text {fit }}$ (Fig. $8 F$ ) was similar to $I_{S T A}^{\text {Peak }}$ (Fig. $1 D$ ).

With multiple linear kernels representing a neuron, as opposed to a simple linear kernel followed by a nonlinearity, this multi-STA LN model incorporates: (1) independent or clustered synaptic plasticity to regulate the strength of inputs impinging on individual dendrite segments (Govindarajan et al., 2006; Govindarajan et al., 2011; Magee, 2011); (2) a linear transformation defined by the STA characterizing that particular dendritic segment (which could be a single oblique or a part of the trunk), with the linear kernel dynamically reconfigurable in response to various properties associated with that dendritic segment; (3) a reconfigurable linear/nonlinear summation that combines the outputs of all these linear kernels; and (4) a somatic nonlinearity, the form of which can also be variable, that transforms these multiple inputs to a single output in the form of a somatic spike (Fig. 8A). Such an approach involving the amalgamation of the roles of synaptic and intrinsic plasticity could enrich current models of learning and memory and be more informative about the roles of single neuron physiology in information encoding and homeostasis (Kim and Linden, 2007; Nelson and Turrigiano, 2008; Sjöström et al., 2008; Remy et al., 2010; Turrigiano, 2011; Narayanan and Johnston, 2012; Honnuraiah and Narayanan, 2013; Ratté et al., 2013).

\section{Discussion}

The primary conclusion of this study is that a single linear kernel is grossly inadequate for defining the spike initiation dynamics of a hippocampal pyramidal neuron that is endowed with extensive dendritic arborization expressing a large array of diverse ion channels. The presence of dendrites and their ion channels alter the spike initiation dynamics in a location-dependent manner, with input statistics and the density, kinetics, and voltage depen-
B

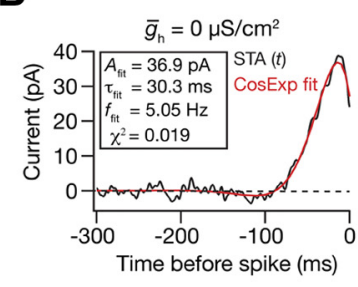

E

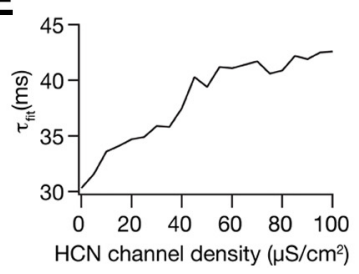

C

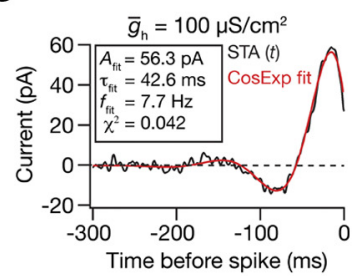

$\mathbf{F}$

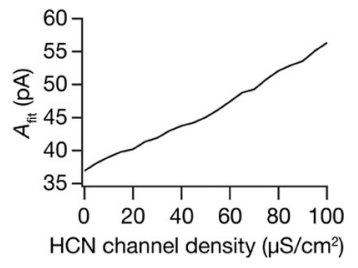

Figure 8. Cosine-modulated exponential serves as a functional form for the quantitative representation of the STA in a multi-

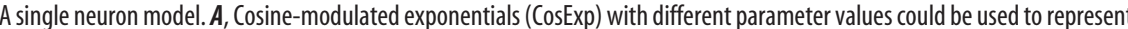

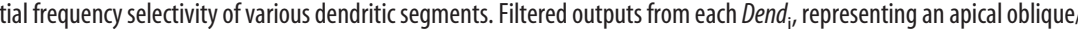
different fit parameters. $\boldsymbol{D}-\boldsymbol{F}$, Evolution of critical fit parameters, the frequency of the sinusoid, $f_{\text {fit }}(\boldsymbol{D})$, the time constant associated

dence of the ion channels playing critical roles in qualitatively and quantitatively altering the nature of the STA. In addition, complex spatiotemporal interdependence of ion channels expressing across the dendritic arbor implies that the impact of the coexistence of several ion channels on the STA could not be inferred by merely understanding the effects of their independent expression. We propose a dynamically reconfigurable multi-STA model as an alternative to the traditional single-STA model for characterizing the input-output relationship of a neuron with plastic active dendrites.

\section{Biophysics of frequency selectivity in neuronal spike initiation dynamics}

An excitability-based taxonomy of neurons divides them into three classes (Hodgkin, 1948), with specific firing rate dependence on the injected current. It has been shown that these three classes of neurons exhibit specific forms of STA, forming an integrator-coincidence detector (I-CD) continuum based on the balance between the net fast inward current and the net slow outward current (Prescott et al., 2008a; Ratté et al., 2013). Our results suggest that variable expression levels in a single ion channel (the HCN channel) can switch the spike initiation dynamics back and forth between an integrator and a coincidence detector (Figs. 1, 2, 3, 4, 5, 6), thereby letting neurons endowed with the HCN channels dynamically span the I-CD continuum. In the frequency domain, although an integrator translates to a lowpass STA (STA characteristic frequency $f_{\mathrm{STA}} \approx 0$ ), a coincidence detector translates to a band-pass STA (Ratté et al., 2013). Our results suggest that the $f_{\mathrm{STA}}$ of a band-pass STA (for inputs impinging on a dendritic location) is strongly related to the subthreshold transfer resonance frequency $\left(f_{\mathrm{TR}}\right)$ of the neuronal compartment where the inputs impinge (Figs. 5, 6, 7). In the case of neurons with active dendrites, the propagation of signals recruits ion channels at the location of input and along the path toward the soma and these ion channels are equipped to modify the STA by altering the balance between the fast and the slow currents. Given the strong relationship between transfer impedance and STA (Figs. 5, 6, 7, Tables 2, 3, 4), the two measurements 
that account for the recruitment of these channels in quantifying location-dependent signal transfer and neuronal gain, these quantities could be used as experimental handles toward understanding the biophysics of neuronal feature selectivity spanning subthreshold and suprathreshold regimes. In addition, the physiological relevance of $f_{\mathrm{STA}}$ as a measure of spectral selectivity in single neuron response dynamics is also emphasized by the strong relationship between $f_{\mathrm{STA}}$ and the suprathreshold resonance frequency $\left(f_{\mathrm{SR}}\right)$ across models (Figs. $\left.1 H-J, 7 H-K\right)$. The dependencies of $f_{\mathrm{SR}}$, a response-based measure of spectral selectivity, on dendritic location and input amplitude (Fig. $7 \mathrm{H}, \mathrm{I}$ ) and its normalization across locations (Fig. $7 \mathrm{~J}, K$ ) serve as additional validation of similar properties in the kernel-based $f_{\mathrm{STA}}$. Collectively, the strong relationships between the three measures of location-dependent spectral selectivity $\left(f_{\mathrm{STA}}, f_{\mathrm{TR}}\right.$ and $f_{\mathrm{SR}} ;$ Fig. $7 \mathrm{~K}$ ) have significant ramifications for how neurons process oscillatory inputs originating at different dendritic locations and for gain modulation through active dendritic mechanisms.

However, STA could still express a weak spectral selectivity even in the absence of subthreshold resonance (Figs. 3, 4). Analyzing the basis for this selectivity, we found a strong relationship between $f_{\text {STA }}$ and the fAHP amplitude (Fig. 3). The fAHP amplitude increased with an increase in either the sodium or the KDR channels, but did not translate to changes in $f_{\mathrm{STA}}$ if the sodium channels recovered slowly from inactivation, pointing to specific roles for such slow recovery in hippocampal dendrites (Colbert et al., 1997). Therefore, although the fAHP amplitude is strongly correlated to $f_{\mathrm{STA}}$, its presence alone is not sufficient to confer spectral selectivity upon spike initiation dynamics. Albeit being a correlative inference from our results, future studies could use ion channels involved in spike repolarization and fAHP differences between pyramidal and interneurons as instruments with which to understand the relationship between spike repolarization and initiation dynamics from a neural coding perspective (Higgs and Spain, 2009; Famulare and Fairhall, 2010; Kispersky et al., 2012; Mease et al., 2013). Finally, our study also highlights the utility of spectral and spectrotemporal analyses in understanding the physiological roles of ion channels and their interactions. Future studies could expand on such analyses by incorporating elements from the sensory literature, such as the spectrotemporal receptive field (Aertsen and Johannesma, 1981; Klein et al., 2006; Sadagopan and Wang, 2009), toward understanding the differential roles of dendritic VGICs and their complex interactions (Rathour and Narayanan, 2012b) in altering a neuron's spectrotemporal response structure.

Plastic active dendrites and neural encoding of input features It has been shown that neurons of different excitability classes exhibit specific forms of STA, forming an I-CD continuum (Ratté et al., 2013). In a neuron endowed with active dendrites, our results imply that the I-CD continuum represented by different forms of STAs could reside within a single neuron, depending on the dendritic location of the impinging input, the input strength, and the spatially heterogeneous intrinsic properties of the dendrite. Because intrinsic properties at a dendritic location are largely governed by the VGICs expressed around that location (Narayanan and Johnston, 2012; Rathour and Narayanan, 2012a), this implies that the well established dendritic gradients in VGIC properties and their localized or global plasticity would govern the specific form of the STA within the I-CD continuum. In conjunction with the state and input dependence of the STA (Figs. 1, 2, 3), this location dependence translates to the ability of a single neuron to function either as an integrator or a coinci- dence detector depending on a specific task. Therefore, it is critical to understand that different dendritic regions of a neuron might be operating within different realms of the I-CD continuum at the same time.

What does location dependence mean for the features to which neuronal spikes are selective? The STA computed using a single GWN input at a given dendritic location is equivalent to studying its spiking dynamics in response to multiple synchronous inputs converging on that dendritic location. Against such equivalence, spectral selectivity in the STA implies that inputs onto a dendritic location have a higher probability of eliciting a somatic spike if they are synchronized to the $f_{\mathrm{STA}}$ for that location. Our results suggest that the location dependence of $f_{\mathrm{STA}}$ is consequent to active and passive filtering by the dendritic arbor, including contributions from the initiation and successful dendrosomatic propagation of local dendritic spikes (Figs. 4, 6, 7). Such location dependence of $f_{\mathrm{STA}}$ implies that different parts of the neuron are optimally tuned to different frequencies, thereby providing a single neuron the computational power to segregate its inputs into different anatomical sections with each tuned to different input features (Fig. 8A). Under such a setup, localized plasticity in dendritic ion channels would translate to changes in the feature selectivity of selected dendritic segments without altering the others, whereas global plasticity would scale up $f_{\text {STA }}$ across the entire dendritic arbor (Figs. $5 G, 6 C$ ). In conjunction with synaptic plasticity, such plasticity in dendritic ion channels provides a neuron with an enormous boost in its information storage capacity (Poirazi and Mel, 2001) whereby a single-neuron can even store specific, correlation-based features (Magee, 2011).

To encompass conclusions from our study, we present a dynamically reconfigurable multi-STA model (Fig. $8 A$ ) as an appropriate representation for a single neuron with plastic active dendrites. Although this model is conceptually similar to the two-layer network model (Poirazi et al., 2003), it encompasses abstractions of specific aspects of the location-dependent spectrotemporal response dynamics, thereby accounting for neuronal feature selectivity. Future studies could focus on experimentally delineating the roles of different ion channels and interactions among them (Rathour and Narayanan, 2012b) in sculpting specific characteristics of the multiple kernels that define location-dependent spike initiation dynamics (Fig. 8A). These endeavors could focus on classes of dendritic VGICs that govern subthreshold resonance (e.g., $\mathrm{HCN}$ and T-type $\mathrm{Ca}^{2+}$ channels), dendritic spike initiation (e.g., $\mathrm{Na}^{+}, \mathrm{Ca}^{2+}$, and $A$-type $\mathrm{K}^{+}$channels), and spike repolarization kinetics (e.g., KDR- and $\mathrm{Ca}^{2+}$-dependent $\mathrm{K}^{+}$channels), and anatomize their contributions to neuronal feature selectivity.

In summary, in this study, we derived several physiologically relevant measurements to quantify spike initiation dynamics and demonstrated the location dependence of STA in model neurons with plastic active dendrites. These measurements facilitated quantitative assessment of the evolution of the STA with changes in neuronal biophysical properties and revealed several specific relationships of the STA to subthreshold impedance. Based on our results, we propose a dynamically reconfigurable multi-STA model for a single neuron and postulate the emergence of a location-dependent map of the $f_{\mathrm{STA}}$ along the somatodendritic axis of a pyramidal neuron (Narayanan and Johnston, 2012). Future experiments could focus on using the power of spectrotemporal representations of feature selectivity to understand the encoding and learning capabilities of a single neuron with plastic active dendrites under physiological and pathophysiological conditions. 


\section{References}

Aertsen AM, Johannesma PI (1981) The spectro-temporal receptive field: a functional characteristic of auditory neurons. Biol Cybern 42:133-143. CrossRef Medline

Agüera y Arcas B, Fairhall AL (2003) What causes a neuron to spike? Neural Comput 15:1789-1807. CrossRef Medline

Andrasfalvy BK, Magee JC (2001) Distance-dependent increase in AMPA receptor number in the dendrites of adult hippocampal CA1 pyramidal neurons. J Neurosci 21:9151-9159. Medline

Angelo K, London M, Christensen SR, Hausser M (2007) Local and global effects of $\mathrm{I}(\mathrm{h})$ distribution in dendrites of mammalian neurons. J Neurosci 27:8643-8653. CrossRef Medline

Ascoli GA, Donohue DE, Halavi M (2007) NeuroMorpho.Org: a central resource for neuronal morphologies. J Neurosci 27:9247-9251. CrossRef Medline

Badel L, Gerstner W, Richardson MJE (2006) Dependence of the spiketriggered average voltage on membrane response properties. Neurocomputing 69:1062-1065. CrossRef

Bittner KC, Andrasfalvy BK, Magee JC (2012) Ion channel gradients in the apical tuft region of CA1 pyramidal neurons. PLoS One 7:e46652. CrossRef Medline

Buzsáki G (2010) Neural syntax: cell assemblies, synapsembles, and readers. Neuron 68:362-385. CrossRef Medline

Carnevale NT, Hines ML (2006) The NEURON Book. Cambridge, UK: Cambridge UP.

Chander D, Chichilnisky EJ (2001) Adaptation to temporal contrast in primate and salamander retina. J Neurosci 21:9904-9916. Medline

Colbert CM, Magee JC, Hoffman DA, Johnston D (1997) Slow recovery from inactivation of $\mathrm{Na}+$ channels underlies the activity-dependent attenuation of dendritic action potentials in hippocampal CA1 pyramidal neurons. J Neurosci 17:6512-6521. Medline

Cook EP, Guest JA, Liang Y, Masse NY, Colbert CM (2007) Dendrite-tosoma input/output function of continuous time-varying signals in hippocampal CA1 pyramidal neurons. J Neurophysiol 98:2943-2955. CrossRef Medline

Dayan P, Abbott LF (2005) Theoretical neuroscience: computational and mathematical modeling of neural systems. Cambridge, MA: MIT.

Debanne D, Campanac E, Bialowas A, Carlier E, Alcaraz G (2011) Axon physiology. Physiol Rev 91:555-602. CrossRef Medline

Ermentrout GB, Galán RF, Urban NN (2007) Relating neural dynamics to neural coding. Phys Rev Lett 99:248103. CrossRef Medline

Famulare M, Fairhall A (2010) Feature selection in simple neurons: how coding depends on spiking dynamics. Neural Comput 22:581-598. CrossRef Medline

Fan Y, Fricker D, Brager DH, Chen X, Lu HC, Chitwood RA, Johnston D (2005) Activity-dependent decrease of excitability in rat hippocampal neurons through increases in I(h). Nat Neurosci 8:1542-1551. CrossRef Medline

Fleidervish IA, Lasser-Ross N, Gutnick MJ, Ross WN (2010) Na+ imaging reveals little difference in action potential-evoked $\mathrm{Na}+$ influx between axon and soma. Nat Neurosci 13:852-860. CrossRef Medline

Gasparini S, Magee JC (2006) State-dependent dendritic computation in hippocampal CA1 pyramidal neurons. J Neurosci 26:2088-2100. CrossRef Medline

Gasparini S, Migliore M, Magee JC (2004) On the initiation and propagation of dendritic spikes in CA1 pyramidal neurons. J Neurosci 24:1104611056. CrossRef Medline

Govindarajan A, Kelleher RJ, Tonegawa S (2006) A clustered plasticity model of long-term memory engrams. Nat Rev Neurosci 7:575-583. CrossRef Medline

Govindarajan A, Israely I, Huang SY, Tonegawa S (2011) The dendritic branch is the preferred integrative unit for protein synthesis-dependent LTP. Neuron 69:132-146. CrossRef Medline

Grossman A, Morlet J (1984) Decomposition of Hardy functions into square integrable wavelet of constant shape. SIAM J Math Anal 15:723736. CrossRef

Higgs MH, Spain WJ (2009) Conditional bursting enhances resonant firing in neocortical layer 2-3 pyramidal neurons. J Neurosci 29:1285-1299. CrossRef Medline

Hodgkin AL (1948) The local electric changes associated with repetitive action in a non-medullated axon. J Physiol 107:165-181. Medline

Hoffman DA, Magee JC, Colbert CM, Johnston D (1997) K+ channel reg- ulation of signal propagation in dendrites of hippocampal pyramidal neurons. Nature 387:869-875. CrossRef Medline

Hong S, Agüera y Arcas B, Fairhall AL (2007) Single neuron computation: from dynamical system to feature detector. Neural Comput 19:31333172. CrossRef Medline

Hong S, Ratté S, Prescott SA, De Schutter E (2012) Single neuron firing properties impact correlation-based population coding. J Neurosci 32: 1413-1428. CrossRef Medline

Honnuraiah S, Narayanan R (2013) A calcium-dependent plasticity rule for HCN channels maintains activity homeostasis and stable synaptic learning. PLoS One 8:e55590. CrossRef Medline

Hu H, Vervaeke K, Graham LJ, Storm JF (2009) Complementary theta resonance filtering by two spatially segregated mechanisms in CA1 hippocampal pyramidal neurons. J Neurosci 29:14472-14483. CrossRef Medline

Hutcheon B, Miura RM, Puil E (1996) Models of subthreshold membrane resonance in neocortical neurons. J Neurophysiol 76:698-714. Medline

Johnston D, Narayanan R (2008) Active dendrites: colorful wings of the mysterious butterflies. Trends Neurosci 31:309-316. CrossRef Medline

Kim SJ, Linden DJ (2007) Ubiquitous plasticity and memory storage. Neuron 56:582-592. CrossRef Medline

Kispersky TJ, Fernandez FR, Economo MN, White JA (2012) Spike resonance properties in hippocampal O-LM cells are dependent on refractory dynamics. J Neurosci 32:3637-3651. CrossRef Medline

Klein DJ, Simon JZ, Depireux DA, Shamma SA (2006) Stimulus-invariant processing and spectrotemporal reverse correlation in primary auditory cortex. J Comput Neurosci 20:111-136. CrossRef Medline

Lai HC, Jan LY (2006) The distribution and targeting of neuronal voltagegated ion channels. Nat Rev Neurosci 7:548-562. CrossRef Medline

Le Van Quyen M, Bragin A (2007) Analysis of dynamic brain oscillations: methodological advances. Trends Neurosci 30:365-373. CrossRef Medline

Lisman JE, Jensen O (2013) The theta-gamma neural code. Neuron 77: 1002-1016. CrossRef Medline

Lörincz A, Notomi T, Tamás G, Shigemoto R, Nusser Z (2002) Polarized and compartment-dependent distribution of $\mathrm{HCN} 1$ in pyramidal cell dendrites. Nat Neurosci 5:1185-1193. CrossRef Medline

Losonczy A, Magee JC (2006) Integrative properties of radial oblique dendrites in hippocampal CA1 pyramidal neurons. Neuron 50:291-307. CrossRef Medline

Magee JC (1998) Dendritic hyperpolarization-activated currents modify the integrative properties of hippocampal CA1 pyramidal neurons. J Neurosci 18:7613-7624. Medline

Magee JC (2000) Dendritic integration of excitatory synaptic input. Nat Rev Neurosci 1:181-190. CrossRef Medline

Magee JC (2011) Observations on clustered synaptic plasticity and highly structured input patterns. Neuron 72:887-888. CrossRef Medline

Magee JC, Cook EP (2000) Somatic EPSP amplitude is independent of synapse location in hippocampal pyramidal neurons. Nat Neurosci 3:895903. CrossRef Medline

Magee JC, Johnston D (1995) Characterization of single voltage-gated $\mathrm{Na}+$ and $\mathrm{Ca}^{2+}$ channels in apical dendrites of rat CA1 pyramidal neurons. J Physiol 487:67-90. Medline

Magee JC, Johnston D (2005) Plasticity of dendritic function. Curr Opin Neurobiol 15:334-342. CrossRef Medline

Mease RA, Famulare M, Gjorgjieva J, Moody WJ, Fairhall AL (2013) Emergence of adaptive computation by single neurons in the developing cortex. J Neurosci 33:12154-12170. CrossRef Medline

Megías M, Emri Z, Freund TF, Gulyás AI (2001) Total number and distribution of inhibitory and excitatory synapses on hippocampal CA1 pyramidal cells. Neuroscience 102:527-540. CrossRef Medline

Migliore M, Shepherd GM (2002) Emerging rules for the distributions of active dendritic conductances. Nat Rev Neurosci 3:362-370. CrossRef Medline

Migliore M, Hoffman DA, Magee JC, Johnston D (1999) Role of an A-type $\mathrm{K}+$ conductance in the back-propagation of action potentials in the dendrites of hippocampal pyramidal neurons. J Comput Neurosci 7:5-15. CrossRef Medline

Narayanan R, Johnston D (2007) Long-term potentiation in rat hippocampal neurons is accompanied by spatially widespread changes in intrinsic oscillatory dynamics and excitability. Neuron 56:1061-1075. CrossRef Medline 
Narayanan R, Johnston D (2008) The h channel mediates location dependence and plasticity of intrinsic phase response in rat hippocampal neurons. J Neurosci 28:5846-5860. CrossRef Medline

Narayanan R, Johnston D (2012) Functional maps within a single neuron. J Neurophysiol 108:2343-2351. CrossRef Medline

Nelson SB, Turrigiano GG (2008) Strength through diversity. Neuron 60: 477-482. CrossRef Medline

Nusser Z (2012) Differential subcellular distribution of ion channels and the diversity of neuronal function. Curr Opin Neurobiol 22:366-371. CrossRef Medline

Poirazi P, Mel BW (2001) Impact of active dendrites and structural plasticity on the memory capacity of neural tissue. Neuron 29:779-796. CrossRef Medline

Poirazi P, Brannon T, Mel BW (2003) Pyramidal neuron as two-layer neural network. Neuron 37:989-999. CrossRef Medline

Poolos NP, Migliore M, Johnston D (2002) Pharmacological upregulation of h-channels reduces the excitability of pyramidal neuron dendrites. Nat Neurosci 5:767-774. CrossRef Medline

Prescott SA, De Koninck Y, Sejnowski TJ (2008a) Biophysical basis for three distinct dynamical mechanisms of action potential initiation. PLoS Comput Biol 4:e1000198. CrossRef Medline

Prescott SA, Ratté S, De Koninck Y, Sejnowski TJ (2008b) Pyramidal neurons switch from integrators in vitro to resonators under in vivo-like conditions. J Neurophysiol 100:3030-3042. CrossRef Medline

Pyapali GK, Sik A, Penttonen M, Buzsáki G, Turner DA (1998) Dendritic properties of hippocampal CA1 pyramidal neurons in the rat: intracellular staining in vivo and in vitro. J Comp Neurol 391:335-352. CrossRef Medline

Rall W (1977) Core conductor theory and cable properties of neurons. In: Handbook of physiology: the nervous system. Cellular biology of neurons (Kandel ER, ed), pp 39-97. Bethesda, MD: American Physiological Society.

Rathour RK, Narayanan R (2012a) Influence fields: a quantitative framework for representation and analysis of active dendrites. J Neurophysiol 107:2313-2334. CrossRef Medline

Rathour RK, Narayanan R (2012b) Inactivating ion channels augment ro- bustness of subthreshold intrinsic response dynamics to parametric variability in hippocampal model neurons. J Physiol 590:5629-5652. CrossRef Medline

Ratté S, Hong S, De Schutter E, Prescott SA (2013) Impact of neuronal properties on network coding: roles of spike initiation dynamics and robust synchrony transfer. Neuron 78:758-772. CrossRef Medline

Remy S, Beck H, Yaari Y (2010) Plasticity of voltage-gated ion channels in pyramidal cell dendrites. Curr Opin Neurobiol 20:503-509. CrossRef Medline

Rieke F, Warland D, van Steveninck R, Bialek W (1999) Spikes: exploring the neural code. Cambridge, MA: MIT.

Sadagopan S, Wang X (2009) Nonlinear spectrotemporal interactions underlying selectivity for complex sounds in auditory cortex. J Neurosci 29:11192-11202. CrossRef Medline

Shah MM, Hammond RS, Hoffman DA (2010) Dendritic ion channel trafficking and plasticity. Trends Neurosci 33:307-316. CrossRef Medline

Sjöström PJ, Rancz EA, Roth A, Häusser M (2008) Dendritic excitability and synaptic plasticity. Physiol Rev 88:769-840. CrossRef Medline

Spruston N (2008) Pyramidal neurons: dendritic structure and synaptic integration. Nat Rev Neurosci 9:206-221. CrossRef Medline

Torrence C, Compo GP (1998) A practical guide to wavelet analysis. Bulletin of the American Meteorological Society 79:61-78. CrossRef

Turrigiano G (2011) Too many cooks? Intrinsic and synaptic homeostatic mechanisms in cortical circuit refinement. Annu Rev Neurosci 34:89103. CrossRef Medline

Ulrich D (2002) Dendritic resonance in rat neocortical pyramidal cells. J Neurophysiol 87:2753-2759. Medline

Vaidya SP, Johnston D (2013) Temporal synchrony and gamma-to-theta power conversion in the dendrites of CA1 pyramidal neurons. Nat Neurosci 16:1812-1820. CrossRef Medline

Williams SR, Stuart GJ (2003) Role of dendritic synapse location in the control of action potential output. Trends Neurosci 26:147-154. CrossRef Medline

Yu Y, Lee TS (2003) Dynamical mechanisms underlying contrast gain control in single neurons. Phys Rev E Stat Nonlin Soft Matter Phys 68:011901. CrossRef Medline 